\title{
An Analysis of the Discontinuous Galerkin Method for a Scalar Hyperbolic Equation
}

\author{
By C. Johnson and J. Pitkäranta
}

\begin{abstract}
We prove $L_{p}$ stability and error estimates for the discontinuous Galerkin method when applied to a scalar linear hyperbolic equation on a convex polygonal plane domain. Using finite element analysis techniques, we obtain $L_{2}$ estimates that are valid on an arbitrary locally regular triangulation of the domain and for an arbitrary degree of polynomials. $L_{p}$ estimates for $p \neq 2$ are restricted to either a uniform or piecewise uniform triangulation and to polynomials of not higher than first degree. The latter estimates are proved by combining finite difference and finite element analysis techniques.
\end{abstract}

1. Introduction. In this note we prove stability and error estimates for the discontinuous Galerkin method applied to the scalar linear hyperbolic model problem

$$
\left\{\begin{array}{l}
u_{\beta}+a u=f \text { in } \Omega, \\
u=g \text { on } \Gamma_{-},
\end{array}\right.
$$

where $\Omega$ is a bounded convex plane domain, $\beta=\left(\beta_{1}, \beta_{2}\right)$ is a constant unit vector, $u_{\beta}=\beta \cdot \nabla u, a$ is a bounded measurable function on $\Omega$, and $\Gamma_{-}$denotes the "inflow" part of the boundary $\partial \Omega: \Gamma_{-}=\{x \in \partial \Omega: \nu(x) \cdot \beta<0\}$, where $\nu(x)$ is the outward unit normal to $\partial \Omega$ at $x$. Let us recall the definition of the discontinuous Galerkin method for (1.1) (cf., [9]). Given a finite element partitioning $\mathscr{C}^{h}=\{T\}$ of $\Omega$, let $P_{k}(T)$ denote the space of polynomials of degree $\leqslant k$ on $T \in \mathscr{C}^{h}$, and seek a function $u_{h}$ defined on $\Omega$ such that for all $T \in \mathscr{C}^{h}, u_{h \mid T} \in P_{k}(T)$ and

$$
\int_{T}\left(u_{h \beta}+a u_{h}\right) v d x+\int_{\partial T_{-}}|\nu \cdot \beta|\left(u_{h}^{+}-u_{h}^{-}\right) v d s=0, \quad v \in P_{k}(T)
$$

where $\nu$ denotes the outward unit normal to $\partial T, \partial T_{-}=\{x \in \partial T: \nu(x) \cdot \beta<0\}$, and $u_{h}^{ \pm}(x)=\lim _{\varepsilon \rightarrow 0^{ \pm}} u_{h}(x+\varepsilon \beta)$, with $u_{h}^{-}(x)=g(x)$ if $x \in \Gamma_{\text {. }}$. As will be seen below, $u_{h}$ is uniquely determined by (1.2) and it is possible to compute $u_{h}$ successively on each $T \in \mathscr{C}_{h}$ starting at the inflow boundary $\Gamma_{-}$where $u_{h}^{-}$is given [9]. Thus, (1.2) is an essentially explicit scheme for (1.1).

The subsets $T$ in (1.2) are usually triangles or quadrilaterals with possibly curved sides on $\partial \Omega$. Here, we assume for simplicity that $\Omega$ is a polygon and that all the subdomains in $\mathscr{C}^{h}$ are triangles. In this form, the scheme (1.2) has been used successfully for solving the neutron transport equation approximately, cf., [11].

Received April 23, 1984.

1980 Mathematics Subject Classification. Primary 65N30. 
To be able here to easily present our results and compare with previous work, let us for simplicity assume that $\Omega$ is the unit square $I \times I$ with $I=(0,1), \beta_{1} \geqslant 0$, $\beta_{2} \geqslant 0$, and that $\mathscr{C}_{h}$ is a uniform triangulation of $\Omega$ with nodes (ih, jh), $0 \leqslant i$, $j \leqslant N=h^{-1}$, where $h$ is the mesh length. Given a piecewise smooth function $v$ on $\Omega$ write $v^{n}(\cdot)=v^{-}(\cdot, n h)$. We shall under various assumptions prove error estimates of the form

$$
\begin{gathered}
\left\|u-u_{h}\right\|_{L_{p}(\Omega)} \leqslant C h^{k+1 / 2}|u|_{W_{p}^{k+1}(\Omega)}, \quad 1 \leqslant p \leqslant \infty \\
\left\|u-u_{h}\right\|_{L_{p}(\Omega)}+\max _{n}\left\|u^{n}-u_{h}^{n}\right\|_{L_{p}(I)} \\
\leqslant C h^{k+\min (1 / 2,1-1 / p)}|u|_{W_{p}^{k+1}(\Omega)}, \quad 1 \leqslant p \leqslant \infty, \\
\max _{n}\left\|u^{n}-u_{h}^{n}\right\|_{L_{p}(I)} \leqslant C h^{k+1 / 2}\left[|u|_{W_{p}^{k+1}(\Omega)}+\max _{n}\left|u^{n}\right|_{W_{p}^{k+1}(I)}\right],
\end{gathered}
$$$$
1 \leqslant p \leqslant 2 .
$$

For $p=2$ we prove analogues of $(1.3 \mathrm{a}, \mathrm{b})$ on general meshes and arbitrary $k \geqslant 0$ using finite element techniques. For $p \neq 2$ and $k=0$ or $k=1$ we prove $(1.3 \mathrm{~b}, \mathrm{c})$ with $1 \leqslant p \leqslant \infty$ and (1.3a) with $2 \leqslant p \leqslant \infty$ on piecewise uniform meshes using a combination of techniques from Fourier and finite element analysis and finally in a similar way we prove (1.3a) for $1 \leqslant p \leqslant 2$ and $k=0,1$ on uniform meshes.

Notice that if $1 \leqslant p \leqslant 2$, then (1.3b) is an optimal estimate in the sense that the exponent of $h$ cannot be increased while keeping the norm on $u$, nor can the regularity requirements on $u$ be weakened while keeping the exponent of $h$. On the other hand, (1.3a) is not optimal in this sense since for the interpolation error $u-\tilde{u}_{h}$, where $\tilde{u}_{h \mid T} \in P_{k}(T), T \in \mathscr{C}_{h}$, is a suitable interpolant of $\left.u\right|_{T}$, we have

$$
\left\|u-\tilde{u}_{h}\right\|_{L_{p}(\Omega)} \leqslant C h^{k+1}|u|_{W_{p}^{k+1}(\Omega)} .
$$

Most likely, the estimate (1.3a) cannot be improved in the above sense for the method (1.2) and it is an open problem if there are other methods for (1.1) which allow such an improvement.

Estimates of the form $(1.3 \mathrm{a}-\mathrm{c})$ for $p=2$ on general meshes were proved in [6], [10] for the so-called streamline diffusion method. In fact, the discontinuous Galerkin method and the streamline diffusion method have very similar properties when applied to (1.1), and the analysis of the two methods is also similar. In particular, it is possible to prove localization results and local error estimates for the discontinuous Galerkin method which are analogous to those presented in [6], [10]. Let us also mention that the $L_{2}$-analysis of both the streamline diffusion method and the discontinuous Galerkin method can be extended to Friedrichs systems, see [6], [8].

The error estimates (1.3) for $p=2$ (and the localization results mentioned above) are based on a stability estimate for (1.2) of the form

$$
\left|u_{h}\right|_{h, \beta}+\left\|u_{h}\right\|_{L_{2}(\Omega)} \leqslant C\left[\|f\|_{L_{2}(\Omega)}+\|g\|_{L_{2}\left(\Gamma_{-}\right)}\right]
$$

where $|\cdot|_{h, \beta}$ is a mesh-dependent seminorm which controls the derivative $u_{h \beta}$ and the jumps of $\nu \cdot \beta u_{h}$ across the interelement boundaries. The stability estimate (1.5) is a discrete (weak) counterpart of the stability inequality

$$
\left\|u_{\beta}\right\|_{L_{2}(\Omega)}+\|u\|_{L_{2}(\Omega)} \leqslant C\left[\|f\|_{L_{2}(\Omega)}+\|g\|_{L_{2}\left(\Gamma_{-}\right)}\right],
$$

which obviously holds for the continuous problem. 
Lesaint and Raviart [9], who gave the first analysis of the discontinuous Galerkin method, proved the following estimate for (1.2) on general meshes for $k \geqslant 0$,

$$
\left\|u-u_{h}\right\|_{L_{2}(\Omega)} \leqslant C h^{k}|u|_{W_{2}^{k+1}(\Omega)} .
$$

Notice that in (1.6) the gap, i.e., the difference between the number of derivatives of $u$ and the exponent of $h$, is equal to one. Results of this type are typical in the usual finite element analysis of linear hyperbolic problems which is based on weaker stability estimates of the form

$$
\left\|u_{h}\right\|_{L_{2}(\Omega)} \leqslant C\left(\|f\|_{L_{2}(\Omega)}+\|g\|_{L_{2}\left(\Gamma_{-}\right)}\right) \text {. }
$$

The estimate (1.3a) with $p=2$, for which the gap is only $\frac{1}{2}$, was used in a crucial way in [7] where an $L_{2}$-analysis of a fully discrete scheme for neutron transport in cylindrical geometry was given. The estimate (1.3a) with $p \neq 2$ may be used to generalize this analysis to $L_{p}, p \neq 2$. Of particular interest (for eigenvalue problems) would then be the case $p=1$. Unfortunately, we have been able to prove (1.3a) for $p=1$ only on a uniform mesh.

The estimates $(1.3 \mathrm{~b}, \mathrm{c})$ are of interest when we consider (1.1) as a model for a linear hyperbolic initial-boundary value problem with $x_{2}$ representing a time variable and where the approximate solution $u_{h}$ is computed successively on the strips $S_{n}=\left\{x \in \Omega:(n-1) h<x_{2} \leqslant n h\right\}, n=1, \ldots, N$, so that $\left\|u^{n}-u_{h}^{n}\right\|_{L_{p}(I)}$ is the error on each time level $x_{2}=n h$. For conventional finite element methods (not including the streamline diffusion method) for (1.1) with piecewise polynomials of degree $k$, the typical result for $p=2$ reads

$$
\max _{n}\left\|u^{n}-u_{h}^{n}\right\|_{L_{2}(I)} \leqslant C h^{k}|u|_{W_{2}^{k+1}(\Omega)}
$$

with a loss of a factor $h^{1 / 2}$ as compared with (1.3b). For (dissipative) finite difference methods a typical result for $(1.1)$ (again with gap $=1$ ) obtained by Fourier methods in the case of a uniform mesh reads

$$
\max _{n}\left\|u^{n}-u_{h}^{n}\right\|_{L_{p}} \leqslant C h^{m}\|g\|_{W_{p}^{m+1}(\mathbf{R})}
$$

where now $\Omega=\left\{x \in \mathbf{R}^{2}: 0<x_{2}<1,-\infty<x_{1}<\infty\right\}, f=0, a=0$, the initial data $g$ is given at $x_{2}=0$, and the order of accuracy of the difference scheme is $m$. One can verify for $k=0$ and $k=1$ that the discontinuous Galerkin method in these cases corresponds to such a dissipative finite difference method of order $m=2 k+1$ (with a special choice of initial data for $k=1$ ), and thus we may by interpolation from (1.7) obtain the result

$$
\max _{n}\left\|u^{n}-u_{h}^{n}\right\|_{L_{p}} \leqslant C h^{k+1 / 2}\|g\|_{W_{p}^{k+1}(\mathbf{R})}
$$

which is the same as $(1.3 \mathrm{~b}, \mathrm{c})$ in the present situation.

The plan of the present paper is as follows. In Section 2 we introduce the notation, prove a basic local stability estimate for the scheme (1.2), and carry out the error analysis in $L_{2}$. Sections 3 and 4 are devoted to the $L_{p}$ stability and error analysis. First, in Section 3, we carry out a Fourier analysis of a finite difference scheme associated with (1.2) in the case where $k=1, a=f=0, \Omega$ is a half-plane, and $\mathscr{C}^{h}$ is a uniform triangulation of $\Omega$. Finally, in Section 4 , we prove $L_{p}$ error estimates using the results of Section 3. 
2. Preliminaries and $L_{2}$-Analysis. For $\Omega$ a convex polygonal plane domain, let $\mathscr{E}$ be a given family of triangulations of $\Omega$ indexed by a parameter $h$ such that if $\mathscr{C}^{h} \in \mathscr{E}$ then $h=\max _{T \in \mathscr{C}^{h}} h_{T}$, where $h_{T}$ denotes the diameter of $T$. For convenience, we assume the geometry of the triangulations to be such that if $T_{1}, T_{2} \in \mathscr{C}^{h} \in \mathscr{E}$, $T_{1} \neq T_{2}$, and $\partial T_{1} \cap \partial T_{2}$ is nonempty then $\partial T_{1} \cap \partial T_{2}$, is either a common side or a common vertex of $T_{1}$ and $T_{2}$. In the analysis below we will further assume that the triangulations are either locally quasi-uniform (Section 2), uniform or piecewise uniform (Sections 3 and 4). The family $\mathscr{E}$ is called locally quasi-uniform if there is a positive constant $\kappa$ such that if $T \in \mathscr{C}^{h} \in \mathscr{E}$, then the angles of $T$ are bounded from below by $\kappa$. In a uniform triangulation $\mathscr{C}^{h}$, all the triangles are identical up to translation and rotation. Finally, $\mathscr{E}$ is called a piecewise uniform triangulation generated by a triangulation $\mathscr{C}$, if any $\mathscr{C}^{h}$ is a refinement of $\mathscr{C}$ such that the restriction of $\mathscr{C}^{h}$ to any $T \in \mathscr{C}$ defines a uniform triangulation of $T$.

In what follows, we use the spaces $L_{p}(\Omega)$ and the Sobolev spaces $W^{m, p}(\Omega)$ and $H^{m}(\Omega)=W^{m, 2}(\Omega), m \geqslant 1,1 \leqslant p \leqslant \infty$, in their usual meaning for $\Omega$ a domain in $\mathbf{R}^{2}$. The norm in $L_{p}(\Omega)$ is denoted by $\|\cdot\|_{p, \Omega}$ if $p \neq 2$ and by $\|\cdot\|_{\Omega}$ if $p=2$. Similarly, if $\Gamma$ is a piecewise smooth curve or a union of such curves, $\|\cdot\|_{p, \Gamma}$ denotes the norm in $L_{p}(\Gamma)$, with the subindex $p$ omitted if $p=2$. Some further meshdependent norms will be introduced later on. Below, we denote by $C$ or $c$, a positive constant which may take different values on different occurrences. The constant may depend on the above parameters $\kappa$ and $k$ but not on other parameters, unless indicated explicitly.

As is shown in [9], one can always solve (2.4) successively, triangle by triangle, with $u_{h}^{-}$given on $\partial T_{-}$either by the previously computed values or by the boundary condition. Thus, $u_{h}$ is determined uniquely if (1.2) can be solved locally in each $T \in \mathscr{C}^{h}$ for given $f$ and $u_{h}^{-}$. That this is the case for $h$ small enough is established by the following result:

Lemma 2.1. Assume that $f \in L_{p}(\Omega)$ and $g \in L_{p}\left(\Gamma_{-}\right), 1 \leqslant p \leqslant \infty$ in (1.1). Then there is a positive constant $h_{0}$ (depending on $\kappa$ and $k$ ) such that if $h_{T}\|a\|_{\infty, T}<h_{0}$ for all $T \in \mathscr{C}^{h}$, then $u_{h}$ is determined uniquely by (1.2) and one has for each $T \in \mathscr{C}^{h}$ the local stability estimates

$$
\begin{array}{r}
\left\|u_{h}\right\|_{p, T}+h_{T}^{1 / p}\left\|u_{h}^{+}\right\|_{p, \partial T_{-}}+h_{T}^{1 / p}\left\|u_{h}^{-}\right\|_{p, \partial T_{+}} \\
\leqslant C\left\{h_{T}\|f\|_{p, T}+h_{T}^{1 / p}\left\|\nu \cdot \beta u_{h}^{-}\right\|_{p, \partial T_{-}}\right\}
\end{array}
$$

and

$$
\left\|u_{h \beta}\right\|_{p, T} \leqslant C\left\{h_{T}^{-1+1 / p}\left\|\nu \cdot \beta\left(u_{h}^{+}-u_{h}^{-}\right)\right\|_{p, \partial T_{-}}+\left\|u_{h}\right\|_{p, T}+\|f\|_{p, T}\right\} .
$$

Proof. Let us first note that, by a scaling argument and by the equivalence of norms in a finite-dimensional space, we have for any $w \in P_{k}(T), T \in \mathscr{C}^{h}$, the inequalities

$$
\begin{aligned}
& C^{-1} h_{T}^{1 / 2-2 / p}\|w\|_{p, T}+h^{1 / 2-1 / p}\|w\|_{p, \partial T} \\
& \quad \leqslant\left\{h_{T} \int_{T} w_{\beta}^{2} d x+\int_{\partial T}|\nu \cdot \beta| w^{2} d s\right\}^{1 / 2} \leqslant C h_{T}^{1 / 2-2 / p}\|w\|_{p, T}
\end{aligned}
$$


where $1 \leqslant p \leqslant \infty$ and $C$ depends only on $\kappa$ and $k$. Now consider a given $T \in \mathscr{C}^{h}$, denote $u_{h \mid T}$ by $\phi_{h}, \phi_{h} \in P_{k}(T)$, and set

$$
\ell\left(\phi_{h}, v\right)=\int_{T}\left(\phi_{h \beta}+a \phi_{h}\right) v d x+\int_{\partial T_{-}} \phi_{h}|\nu \cdot \beta| v d s .
$$

Choosing $v=\phi_{h}+\gamma h_{T} \phi_{h \beta}$, where $\gamma \in(0,1]$ is defined below, we have

$$
\|v\|_{p, T} \leqslant C\left\|\phi_{h}\right\|_{p, T}, \quad 1 \leqslant p \leqslant \infty
$$

and

$$
\begin{aligned}
\ell\left(\phi_{h}, v\right)= & \frac{1}{2} \int_{\partial T} \phi_{h}^{2}|\nu \cdot \beta| d s+\gamma h_{T} \int_{T} \phi_{h \beta}^{2} d x \\
& +\gamma h_{T} \int_{\partial T_{-}}|\nu \cdot \beta| \phi_{h} \phi_{h \beta} d s+\int_{T} a \phi_{h}\left(\phi_{h}+\gamma h_{T} \phi_{h \beta}\right) d x .
\end{aligned}
$$

Using here (2.1) and (2.2) and recalling that $\gamma \leqslant 1$ we obtain

$$
\ell\left(\phi_{h}, v\right) \geqslant \frac{\gamma}{4}\left(1-C \gamma-C h_{T}\|a\|_{\infty, T}\right)\left\{h_{T} \int_{T} \phi_{h \beta}^{2} d x+\int_{\partial T} \phi_{h}^{2}|\nu \cdot \beta| d s\right\} .
$$

Choosing here $\gamma=\min \{1,1 / 2 C\}$ and assuming that $C h_{T}\|a\|_{\infty, T} \leqslant \frac{1}{4}$, say, we conclude, recalling (2.1) and (1.2) that for some constant $C$,

$$
\begin{aligned}
& C^{-1}\left\{h_{T}^{1-4 / p}\left\|\phi_{h}\right\|_{p, T}^{2}+h^{1-2 / p}\left\|\phi_{h}\right\|_{p, \partial T}^{2}\right\} \\
& \quad \leqslant b\left(\phi_{h}, v\right)=\int_{T} f v d x+\int_{\partial T_{-}} u_{h}^{-} v|\nu \cdot \beta| d s .
\end{aligned}
$$

Applying on the right-hand side the Hölder inequality, and recalling (2.1) and (2.2), the first inequality in the lemma follows. The second inequality can now be proved analogously, by choosing $v=\phi_{h \beta}$ in (1.2). We omit the details.

In what follows, we associate with each $\mathscr{C}^{h} \in \mathscr{E}$ a finite element space $V_{h}$ defined by

$$
V_{h}=\left\{v \in L_{2}(\Omega):\left.v\right|_{T} \in P_{k}(T), T \in \mathscr{C}^{h}\right\} .
$$

Further, we set

$$
\Gamma_{h}=\left(\bigcup_{T \in \mathscr{C}^{h}} \partial T\right) \backslash \partial \Omega .
$$

If $T \subset \Omega$ and $S \subset \Gamma_{h} \cup \partial \Omega$, we use the abbreviations

$$
(v, w)_{T}=\int_{T} v w d x, \quad\langle v, w\rangle_{S}=\int_{S} v w|\nu \cdot \beta| d s,
$$

where $\nu$ is a normal to $S$. In this section we drop the subindex $T$ if $T=\Omega$. For a piecewise continuous function $v$, we set $v^{ \pm}(x)=\lim _{\varepsilon \rightarrow 0^{ \pm}} v(x \pm \varepsilon \beta)$ for $x \in \Gamma_{h}$.

By summing over $T \in \mathscr{C}^{h}$ in (1.2), we obtain the following equivalent formulation of the discontinuous Galerkin method. Given $k$ and $\mathscr{C}^{h}$, find $u_{h} \in V_{h}$ such that

$$
\mathscr{B}\left(u_{h}, v\right)=(f, v)+\langle g, v\rangle_{\Gamma_{-}}, \quad v \in V_{h},
$$

where

$$
\mathscr{B}(w, v)=\sum_{T \in \mathscr{C}^{h}}\left(w_{\beta}+a w, v\right)_{T}+\left\langle w^{+}-w^{-}, v^{+}\right\rangle_{\Gamma_{h}}+\langle w, v\rangle_{\Gamma_{-}} .
$$


Using the partial integration formula

$$
\left(w_{\beta}, v\right)_{T}=\langle w, v\rangle_{\partial T_{+}}-\langle w, v\rangle_{\partial T_{-}}-\left(w, v_{\beta}\right)_{T}
$$

we can also write

$$
\mathscr{B}(w, v)=\sum_{T \in \mathscr{C}^{h}}\left(w,-v_{\beta}+a v\right)_{T}+\left\langle w^{-}, v^{-}-v^{+}\right\rangle_{\Gamma_{h}}+\langle w, v\rangle_{\Gamma_{+}} .
$$

Note that we can replace $u_{h}$ by the exact solution $u$ in (2.4), i.e., we have the consistency relation

$$
\mathscr{B}\left(u-u_{h}, v\right)=0, \quad v \in V_{h} .
$$

In the error analysis below, we also make use of the following dual variational problem: Given $\varphi \in L_{1}(\Omega)$, find $\phi_{h} \in V_{h}$ such that

$$
\mathscr{B}\left(w, \phi_{h}\right)=(\varphi, w), \quad w \in V_{h} .
$$

In view of (2.6), $\phi_{h}$ is simply the discontinuous Galerkin solution to the problem

$$
\begin{cases}-\phi_{\beta}+a \phi=\varphi & \text { in } \Omega \\ \phi=0 & \text { on } \Gamma_{+} .\end{cases}
$$

Let us now assume that $\mathscr{E}$ is a locally quasi-uniform family of triangulations and let us associate with each $\mathscr{C}^{h} \in \mathscr{E}$ a seminorm $|\cdot|_{h, \beta}$ and a norm $\|\cdot \mid\|_{h, \beta}$ defined by

$$
\begin{gathered}
|v|_{h, \beta}^{2}=\sum_{T \in \mathscr{C}^{h}} h_{T}\left\|v_{\beta}\right\|_{T}^{2}+\left\langle\left\langle v^{+}-v^{-}\right\rangle\right\rangle_{\Gamma_{h}}^{2}+\langle\langle v\rangle\rangle_{\Gamma}^{2}, \\
\|v\|_{h, \beta}^{2}=\|v\|^{2}+|v|_{h, \beta}^{2},
\end{gathered}
$$

where $\langle\langle v\rangle\rangle_{S}^{2}=\langle v, v\rangle_{S}$. Then we can prove the following result, which is one of the main results of this paper.

THEOREM 2.1. Let $\mathscr{E}$ be a locally quasi-uniform family of triangulations of $\Omega$, and let $V_{h}$ be defined by (2.3) for each $\mathscr{C}^{h} \in \mathscr{E}$ and for some given $k \geqslant 0$. Then, there is a constant $h_{0}$ depending on $\kappa$ and $k$ such that if $u$ is the solution to (1.1) and $h^{1 / 2}\|a\|_{\infty, \Omega}<h_{0}$, the solution $u_{h}$ to (2.4) satisfies the stability estimate

$$
\left\|u_{h}\right\|_{h, \beta} \leqslant C\left(\|f\|_{\Omega}+\left\||\nu \cdot \beta|^{1 / 2} g\right\|_{\Gamma_{-}}\right)
$$

and the error estimate

$$
|| u-u_{h}||_{h, \beta} \leqslant C h^{k+1 / 2}|u|_{H^{k+1}(\Omega)},
$$

where the constants depend on $\|a\|_{\infty, \Omega}$ and $\operatorname{diam}(\Omega)$.

Remark. Note that from the error estimate (2.10) it follows, in particular, that

$$
\left\langle\left\langle u-u_{h}\right\rangle\right\rangle_{\Gamma} \leqslant C h^{k+1 / 2}|u|_{H^{k+1}(\Omega)}
$$

or, more generally: we have for any $\Omega^{\prime} \subset \Omega$ with boundary $\Gamma^{\prime}$ such that $\Gamma_{-}^{\prime}=\Gamma_{-}$that

$$
\left\langle\left\langle u-u_{h}\right\rangle\right\rangle_{\Gamma^{\prime}} \leqslant C h^{k+1 / 2}|u|_{H^{k+1}\left(\Omega^{\prime}\right)} \text {. }
$$

This means that the estimate (2.10) is optimal. (See the Introduction.)

Proof. Following [10] we introduce the weight function

$$
\psi(x)=e^{-\gamma\left(x-x_{0}\right) \cdot \beta},
$$


where $x_{0}$ is a point on $\partial \Omega$ chosen so that $\|\psi\|_{\infty, \Omega}=1$. For $\chi \in L_{2}(\Omega)$, let $\tilde{\chi}$ denote the $L_{2}$-projection of $\chi$ into $V_{h}$, i.e., $\tilde{\chi}$ is defined by

$$
(\chi-\tilde{\chi}, v)_{T}=0, \quad v \in P_{k}(T), T \in \mathscr{C}^{h} .
$$

By (2.4), we have

$$
\mathscr{B}\left(u_{h}, \psi u_{h}\right)=\mathscr{B}\left(u_{h}, \psi u_{h}-\widetilde{\psi} u_{h}\right)+\left(f, \widetilde{\psi} u_{h}\right)+\left\langle g, \widetilde{\psi} u_{h}\right\rangle_{\Gamma_{-}} .
$$

On the left-hand side we obtain by straightforward computation

$$
\begin{aligned}
\mathscr{B}\left(u_{h}, \psi u_{h}\right)= & \left(u_{h},\left(\psi a-\frac{1}{2} \psi_{\beta}\right) u_{h}\right) \\
& +\frac{1}{2}\left\langle u_{h}^{+}-u_{h}^{-}, \psi\left(u_{h}^{+}-u_{h}^{-}\right)\right\rangle_{\Gamma_{h}}+\frac{1}{2}\left\langle u_{h}, \psi u_{h}\right\rangle_{\Gamma} .
\end{aligned}
$$

Choosing $\gamma=2\|a\|_{\infty}+1$ and noting that

$$
-\psi_{\beta}(x)=\gamma \psi(x) \geqslant \gamma e^{-\gamma \operatorname{diam}(\Omega)},
$$

we obtain

$$
\mathscr{B}\left(u_{h}, \psi u_{h}\right) \geqslant C\left\{\|u\|^{2}+\frac{1}{2}\left\langle\left\langle u_{h}^{+}-u_{h}^{-}\right\rangle\right\rangle_{\Gamma_{h}}^{2}+\frac{1}{2}\left\langle\left\langle u_{h}\right\rangle\right\rangle_{\Gamma}^{2}\right\},
$$

where $C$ depends on $\gamma$ and $\operatorname{diam} \Omega$. To estimate the right-hand side of (2.11), we use the standard estimates

$$
\begin{gathered}
\|\chi-\tilde{\chi}\|_{T} \leqslant C h_{T}^{k+1}|\chi|_{H^{k+1}(T)}, \\
\|\chi-\tilde{\chi}\|_{\partial T} \leqslant C h_{T}^{k+1 / 2}|\chi|_{H^{k+1}(T)} .
\end{gathered}
$$

Since $\left|u_{h}\right|_{H^{k+1}(T)}=0,\|\psi\|_{W^{\prime, \infty}(T)} \leqslant C \gamma^{l}$ and $\left\|u_{h}\right\|_{H^{l}(T)} \leqslant C h_{T}^{-l}\left\|u_{h}\right\|_{T}, 0 \leqslant l \leqslant k+1$, we obtain

$$
\left\|\psi u_{h}-\widetilde{\psi} u_{h}\right\|_{T}+h_{T}^{1 / 2}\left\|\psi u_{h}-\widetilde{\psi} u_{h}\right\|_{\partial T} \leqslant C \max \left\{\gamma h_{T},\left(\gamma h_{T}\right)^{k+1}\right\}\left\|u_{h}\right\|_{T} .
$$

Now setting $v=\psi u_{h}-\widetilde{\psi u}_{h}$ and recalling that $(v, w)_{T}=0$ for $w \in P_{k}(T), T \in \mathscr{C}^{h}$, we conclude from (2.5) that

$$
\mathscr{B}\left(u_{h}, v\right)=\left\langle u_{h}^{+}-u_{h}^{-}, v^{+}\right\rangle_{\Gamma_{h}}+\left\langle u_{h}, v\right\rangle_{\Gamma_{-}}+\left(a u_{h}, v\right) .
$$

By the above interpolation error estimates, if $\gamma h_{T} \leqslant 1$, we have

$$
\left\langle\left\langle v^{ \pm}\right\rangle\right\rangle_{\Gamma_{h}}+\langle\langle v\rangle\rangle_{\Gamma} \leqslant C \gamma h^{1 / 2}\left\|u_{h}\right\|, \quad\|v\| \leqslant C \gamma h\left\|u_{h}\right\|,
$$

so that

$$
\left|\mathscr{B}\left(u_{h}, \psi u_{h}-\widetilde{\psi} u_{h}\right)\right| \leqslant \frac{1}{4}\left(\left\|u_{h}\right\|^{2}+\left\langle\left\langle u_{h}^{+}-u_{h}^{-}\right\rangle\right\rangle_{\Gamma_{h}}^{2}+\left\langle\left\langle u_{h}\right\rangle\right\rangle_{\Gamma}^{2}\right)+C \gamma^{2} h\left\|u_{h}\right\|^{2} .
$$

Assuming now that $\gamma h^{1 / 2}=\left(2\|a\|_{\infty}+1\right) h^{1 / 2}$ is small enough, and combining (2.11), (2.12) and Lemma 2.1, we obtain

$$
C\left\|u_{h}\right\|_{h, \beta}^{2} \leqslant \mathscr{B}\left(u_{h}, \widetilde{\psi} u_{h}\right) \leqslant C^{\prime}\left(\|f\|+\left\||\nu \cdot \beta|^{1 / 2} g\right\|_{\Gamma_{-}}\right)\left\|u_{h}\right\|_{h, \beta},
$$

which proves the asserted stability estimate.

The error estimate follows in a similar fashion noting that

$$
\begin{aligned}
C\left\|u_{h}-\tilde{u}\right\|_{h, \beta}^{2} & \leqslant \mathscr{B}\left(u_{h}-\tilde{u}, \widetilde{\psi\left(u_{h}-\tilde{u}\right)}\right)=\mathscr{B}\left(u-\tilde{u}, \widetilde{\psi\left(u_{h}-\tilde{u}\right)}\right) \\
& \leqslant C h^{k+1 / 2}|u|_{K^{k+1}(\Omega)}\left\|u_{h}-\tilde{u}\right\|_{h, \beta} .
\end{aligned}
$$

Here the equality is a consequence of (2.7) and the last inequality follows from (2.6) and (2.13). 
3. Finite Difference Analysis. In this section we apply the discontinuous Galerkin method to the simplified model problem: Find the function $u=u(x, t)$ defined on $\mathbf{R} \times \mathbf{R}^{+}$such that

$$
\begin{cases}\frac{\partial u}{\partial t}+\gamma \frac{\partial u}{\partial x}=0, & (x, t) \in \mathbf{R} \times \mathbf{R}^{+}, \\ u(0, x)=g(x) & x \in \mathbf{R} .\end{cases}
$$

This is obviously a special case of problem (1.1) where $\Omega$ is a half-plane, $a=f=0$ and $\gamma=\beta_{2} / \beta_{1}$ is a constant. We assume below that $0 \leqslant \gamma \leqslant 1$. The finite element partitioning $\mathscr{C}^{h}$ of $\Omega=\mathbf{R} \times \mathbf{R}^{+}$is defined in terms of the grid points

We set

$$
\begin{aligned}
& x_{i}=i h, \quad i=0, \pm 1, \pm 2, \ldots, \\
& t_{n}=n h, \quad n=0,1,2, \ldots .
\end{aligned}
$$

$$
\mathscr{C}^{h}=\left\{T_{n i}^{+}, T_{n i}^{-}, n=0,1, \ldots, i=0, \pm 1, \ldots\right\},
$$

where $T_{n i}^{+}$is a triangle with vertices at $\left(x_{i-1}, t_{n-1}\right),\left(x_{i}, t_{n-1}\right)$ and $\left(x_{i}, t_{n}\right)$ and $T_{n i}^{-}$ has its vertices at $\left(x_{i-1}, t_{n-1}\right),\left(x_{i-1}, t_{n}\right)$ and $\left(x_{i}, t_{n}\right)$ (see Figure 1$)$. Now if $u_{h}^{-}\left(\cdot, t_{n-1}\right)$ is given for some $n \geqslant 1$ we can solve (1.2) for $u_{h}$ on any $T=T_{n i}^{+}$and thereafter on any $T=T_{n i}^{-}$, thus obtaining $u_{h}^{-}\left(\cdot, t_{n}\right)$. Denoting by $W_{h}$ the space

$$
W_{h}=\left\{v \in L_{2}(\mathbf{R}): v_{\mid I_{i}} \in P_{k}\left(I_{i}\right), I_{i}=\left(x_{i-1}, x_{i}\right), i=0, \pm 1, \ldots\right\},
$$

we can write the discrete solution algorithm formally as

$$
\begin{gathered}
u_{h}^{-}\left(\cdot, t_{n}\right)=G_{h} u_{h}^{-}\left(\cdot, t_{n-1}\right), \quad n \geqslant 1, \\
u_{h}^{-}(\cdot, 0)=\pi_{h} g,
\end{gathered}
$$

where $G_{h}: W_{h} \rightarrow W_{h}$ is a linear operator independent of $n$, and $\pi_{h}$ denotes the $L_{2}$-projection into $W_{h}$. We have here utilized the fact that the numerical scheme is unchanged if $g$ is replaced by $\pi_{h} g$, a property that is obvious from (1.2).

To see the structure of the operator $G_{h}$ more closely, consider the triangles $T_{n, i-1}^{+}, T_{n i}^{+}$and $T_{n i}^{-}$for some given $n, i$ (see Figure 1). Since $\gamma \in[0,1]$, we can solve (1.2) for $T=T_{n i}^{+}$once $u_{h}^{-}\left(x, t_{n-1}\right)$ is known for $x \in\left(x_{i-1}, x_{i}\right)$. In fact, we obtain for all $i$,

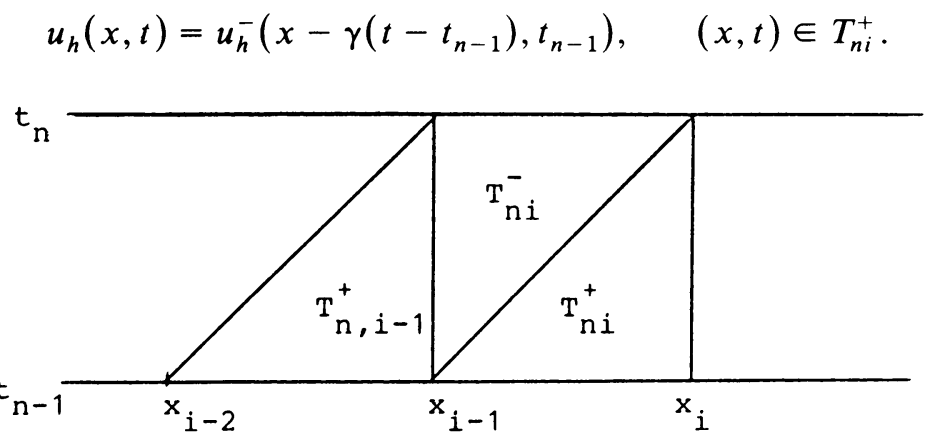

Figure 1

Using (3.3) we can now determine $u_{h}$ on $T_{n i}^{-}$from

$$
\begin{aligned}
& \int_{T_{n i}^{-}}\left(\frac{\partial u_{h}}{\partial t}+\gamma \frac{\partial u_{h}}{\partial x}\right) v d x+\int_{\left(\partial T_{n i}^{-}\right)_{-}}\left(u_{h}^{+}-u_{h}^{-}\right) v \nu \cdot \beta d s=0 \\
& v \in P_{k}\left(T_{n i}^{-}\right) .
\end{aligned}
$$


Let us choose here $v(x, t)=w(x-\gamma t)$, where $w$ is a polynomial of degree $\leqslant k$ on

R. Then $\partial v / \partial t+\gamma \partial v / \partial x=0$ and we obtain by partial integration in (3.4) that

$$
\int_{\left(\partial T_{n i}^{-}\right)_{-}} u_{h}^{-} v \nu \cdot \beta d s=\int_{\left(\mathrm{\partial} T_{n i}^{-}\right)_{+}} u_{h}^{-} v \nu \cdot \beta d s .
$$

Noting that $\nu \cdot \beta d s=\alpha d x$ on $\partial T_{n i}^{+}$with $\alpha$ a constant, and recalling (3.3), we further obtain

$$
\int_{x_{i-1}}^{x_{i}}\left[u_{h}^{-}\left(x, t_{n}\right)-u_{h}^{-}\left(x-\gamma h, t_{n-1}\right)\right] w\left(x-\gamma t_{n}\right) d x=0 .
$$

Since $w$ is here an arbitrary polynomial of degree $\leqslant k$, we conclude that $G_{h}$ is defined by

$$
G_{h}=\pi_{h} G
$$

where $G$ denotes the corresponding exact solution operator:

$$
(G v)(x)=v(x-\gamma h) .
$$

We may interpret (3.2) as a finite difference scheme by introducing the notation

$$
\begin{gathered}
U^{n}(x)=\left[U_{1}^{n}(x), \ldots, U_{k}^{n}(x)\right]^{T}, \\
U_{1}^{n}(x)=u_{h}^{-}\left(x, t_{n}\right), \\
U_{2}^{n}(x)=h \frac{\partial u_{h}^{-}}{\partial x}\left(x, t_{n}\right), \\
\vdots \\
U_{k+1}^{n}(x)=h^{k} \frac{\partial^{k} u_{h}^{-}}{\partial x^{k}}\left(x, t_{n}\right),
\end{gathered}
$$

where $x \in\left\{x_{i+1 / 2}=(i+1 / 2) h, i=0, \pm 1, \ldots\right\}$. Obviously, $U^{n}\left(x_{i+1 / 2}\right)$ defines $u_{h}^{-}\left(\cdot, t_{n}\right)$ uniquely on the interval $\left(x_{i}, x_{i+1}\right)$ and vice versa. Since $u_{h}^{-}\left(\cdot, t_{n}\right)$ is determined uniquely on $\left(x_{i-1}, x_{i}\right)$ when $u_{h}^{-}\left(\cdot, t_{n-1}\right)$ is known on $\left(x_{i-2}, x_{i}\right),(3.2)$ corresponds to a difference scheme of the form

$$
U^{n}(x)=A_{1} U^{n-1}(x)+A_{2} U^{n-1}(x-h) \equiv\left(E U^{n-1}\right)(x), \quad x \in \mathbf{R},
$$

where $A_{1}$ and $A_{2}$ are $(k+1) \times(k+1)$ matrices, and we have made the usual extension of the scheme to all $x \in \mathbf{R}$. The matrices depend only on the parameters $\gamma$ and $k$, so the operator $E$ defined by (3.7) is translation-invariant.

In the case $k=0$ we have

$$
A_{1}=1-\gamma, \quad A_{2}=\gamma,
$$

i.e., (3.7) reduces to the ordinary upwind scheme in this case. If $k=1$, one has

$$
A_{1}=(1-\gamma)\left(\begin{array}{cc}
1 & -\frac{1}{2} \gamma \\
6 \gamma & 1-2 \gamma-2 \gamma^{2}
\end{array}\right), \quad A_{2}=\gamma\left(\begin{array}{cc}
1 & \frac{1}{2}(1-\gamma) \\
-6(1-\gamma) & -3+6 \gamma-2 \gamma^{2}
\end{array}\right) \text {. }
$$

Below we confine our attention to the case $k=1$. We let $S_{n}, n \geqslant 0$, be defined by

$$
S_{n}=\bigcup_{i}\left(\partial T_{n i}^{+}\right)_{+}=\bigcup_{i}\left(\partial T_{n i}^{-}\right)_{-} .
$$


Also, we let $\beta=(1, \gamma)$, so that

$$
|\nu \cdot \beta(x)|= \begin{cases}\gamma, & x \in\left(\partial T_{n i}^{-}\right)_{-} \cap\left(\partial T_{n, i-1}^{+}\right)_{+}, \\ 1-\gamma, & x \in\left(\partial T_{n i}^{-}\right)_{-} \cap\left(\partial T_{n i}^{+}\right)_{+} .\end{cases}
$$

We also introduce the parameter $\rho$ defined by

$$
\rho=\gamma(1-\gamma) \text {. }
$$

The main purpose of this section is to prove the following

TheOREM 3.1. Let $k=1$ and let $u_{h}$ be defined on $\mathbf{R} \times \mathbf{R}^{+}$by (3.2) through (3.6). Then there is a constant $C$ such that for all $n, p, n \geqslant 0,1 \leqslant p \leqslant \infty$,

$$
\left\|u_{h}^{-}\left(\cdot, t_{n}\right)\right\|_{p, \mathbf{R}} \leqslant C\|g\|_{p, \mathbf{R}}
$$

and

$$
\left\|\nu \cdot \beta\left(u_{h}^{+}-u_{h}^{-}\right)\right\|_{p, S_{n}}=C(\rho / n)^{1 / 2}\|g\|_{p, \mathbf{R}} .
$$

The proof of Theorem 3.1 is based on Fourier analysis of the finite difference scheme (3.7) and is split below into several lemmas. We begin by introducing the Fourier transform

$$
\hat{U}^{n}(\xi)=\int_{-\infty}^{\infty} e^{-i x \xi} U^{n}(x) d x, \quad \xi \in \mathbf{R},
$$

which allows (3.7) to be written as

$$
\hat{U}^{n}(\xi)=\hat{E}(h \xi) \hat{U}^{n-1}(\xi)
$$

where $\hat{E}(\theta)=A_{1}+e^{-i \theta} A_{2}$. Below we use a vector norm $|\cdot|$ defined by

$$
|V|=\left(\left|V_{1}\right|^{2}+\frac{1}{12}\left|V_{2}\right|^{2}\right)^{1 / 2}
$$

where $V_{1}$ and $V_{2}$ may be real- or complex-valued. The reason for using this norm, as will be seen in Lemma 3.2 below, is the obvious relation

$$
|V|=\|v\|_{L_{2}(0,1)}, \quad v(x)=V_{1}+V_{2}\left(x-\frac{1}{2}\right), x \in(0,1) .
$$

If $A$ is a $2 \times 2$ matrix, we set

$$
|A|=\sup _{V \neq 0} \frac{|A V|}{|V|} .
$$

Further, if $V=\left(V_{1}, V_{2}\right)^{T}$ is a vector-valued function defined on $S \subset \mathbf{R}$, we denote by $\|V\|_{p, S}$ or by $\|V\|_{L_{p}(S)}$ a norm of $V$ defined by

$$
\|V\|_{p, S}=\|V\|_{L_{p}(S)}=\|V(\cdot) \mid\|_{L_{p}(S)} .
$$

Similarly, if $A$ is a $2 \times 2$ matrix with coefficients real- or complex-valued functions defined on $S \subset \mathbf{R}$, we set

$$
\|A\|_{p, S}=\|A\|_{L_{p}(S)}=\|A(\cdot) \mid\|_{L_{p}(S)} .
$$

Let $\left[L_{p}(\mathbf{R})\right]^{2}$ denote the space of vector-valued functions $V=\left(V_{1}, V_{2}\right)^{T}$ defined on $\mathbf{R}$ such that $V_{i} \in L_{p}(\mathbf{R}), i=1,2$, and let $E$ be a linear translation-invariant operator defined on the whole space $\left[L_{p}(\mathbf{R})\right]^{2}$ for some $p, 1 \leqslant p \leqslant \infty$. Denote by $\|E\|_{p}$ the norm of $E$ defined as

$$
\|E\|_{p}=\sup _{\substack{V \in\left[L_{p}(\mathbf{R})\right]^{2} \\ V \neq 0}} \frac{\|E V\|_{p, \mathbf{R}}}{\|V\|_{p, \mathbf{R}}}
$$


Assume further that the Fourier transform of $E V, V \in\left[L_{p}(\mathbf{R})\right]^{2}$, as defined above, is given by

$$
(\widehat{E V})(\xi)=\hat{E}(h \xi) \hat{V}(\xi), \quad \xi \in \mathbf{R},
$$

where $h>0$ and $\hat{E}(\theta)$ is $2 \pi$-periodic. Note that the operator $E$, defined by (3.7), satisfies these assumptions for any $p$.

The operator norm $\|E\|_{p}$ can be estimated using the following lemma, which states the well-known Carlson-Beurling inequality (cf. [4]). In the lemma, $\chi$ denotes a smooth function defined on $\mathbf{R}$ such that $\chi(\theta)=0$ for $|\theta|>3 \pi / 2$ and $\chi(\theta)=1$ for $|\theta| \leqslant \pi$.

LEMMA 3.1. Under the above assumptions write $\tilde{E}(\theta)=e^{-i \alpha \theta} \hat{E}(\theta)$, where $\alpha \in \mathbf{R}$ is arbitrary. Then $\|E\|_{p}=M_{p}(\tilde{E}(\theta))$, where the latter can be estimated as

$$
M_{p}(\tilde{E}(\theta)) \leqslant C\left\{\|\chi \tilde{E}\|_{L_{2}(\mathbf{R})}\left\|\frac{d}{d \theta}(\chi \tilde{E})\right\|_{L_{2}(\mathbf{R})}\right\}^{1 / 2} .
$$

In order to prove the stability estimate (3.8), we obviously need an estimate for the translation-invariant operator $E^{n}: U^{0} \rightarrow U^{n}$ defined by (3.7). We prepare the situation so that Lemma 3.1 can finally be used. An essential step is then to estimate the growth rate of $\hat{E}(\theta)^{n}=\left(A_{1}+e^{-i \theta} A_{2}\right)^{n}$ as $n$ increases. If $\hat{E}(\theta)$ has two linearly independent eigenfunctions associated with the eigenvalues $\lambda_{1}$ and $\lambda_{2}$, we can use the usual splitting

$$
\hat{E}^{n}(\theta)=D^{-1}(\theta) \Lambda(\theta)^{n} D(\theta), \quad n=1,2, \ldots,
$$

where $\Lambda(\theta)=\operatorname{diag}\left\{\lambda_{1}, \lambda_{2}\right\}$. However, since $\hat{E}(\theta)$ is nonsymmetric and depends on both $\gamma$ and $\theta$, it is difficult to prove the existence of the diagonalizing matrix $D$ in (3.10) in general. Therefore, we use below the splitting (3.10) only for small $|\theta|$ and near the points $(\theta, \gamma)=( \pm \pi, 0)$ and $(\theta, \gamma)=( \pm \pi, 1)$; for the remaining values of the parameters, as it turns out, it suffices to estimate the matrix norm of $\hat{E}(\theta)$.

The essential properties of $\hat{E}(\theta)$ to be required in the subsequent analysis are established by the following three lemmas.

LEMMA 3.2. There is a positive constant $\kappa$ such that $\hat{E}^{n}(\theta)$ admits the representation (3.10) in the range $(\theta, \gamma) \in D_{\kappa}=[-\kappa, \kappa] \times[0,1]$. The eigenvalues of $\hat{E}(\theta)$ are smooth functions of $\gamma$ and $\theta$ in $D_{\kappa}$ and satisfy

$$
\begin{aligned}
& \lambda_{1}=\exp \left\{-i \gamma \theta-a(\gamma) \rho \theta^{4}+O\left(\rho|\theta|^{5}\right)\right\}, \\
& \lambda_{2}=\exp \{-c \rho+O(\rho|\theta|)\},
\end{aligned}
$$

where $a(\gamma)$ is strictly positive for $\gamma \in[0,1]$ and $c$ is a positive constant. Moreover, $|D(\theta)|$ and $\left|D^{-1}(\theta)\right|$ are bounded by a constant for all $(\theta, \gamma) \in D_{\kappa}$.

Proof. For a given $\gamma \in(0,1)$, let $\lambda(\theta)$ be an eigenvalue of $\hat{E}(\theta)$ and write

$$
\lambda(\theta)=e^{-i \gamma \theta}+\rho \xi=(1-\phi)^{\gamma}+\rho \xi=1-\gamma \phi+\rho[\psi(\gamma, \phi)+\xi],
$$

where we have introduced the new variable $\phi=1-e^{-i \theta}$. For any $\gamma \in[0,1], \psi$ admits the series representation

$$
\psi(\gamma, \phi)=-\sum_{k=2}^{\infty} \frac{\Gamma(k-\gamma)}{\Gamma(k+1) \Gamma(2-\gamma)} \phi^{k},
$$


which converges in the open unit disc $|\phi|<1$. Thus $\psi$ is an analytic function of $\phi$ on the open unit disc and so $\psi=\psi(\gamma, \phi(\theta))$ is a smooth function of $\theta$ on the interval $[-c, c]$ for any $\gamma \in[0,1]$ and $c<\pi / 3$.

Denoting by $I$ the identity matrix, we obtain, using the above notation,

$$
\hat{E}(\theta)-\lambda(\theta) I=\rho\left(\begin{array}{cc}
\psi(\gamma, \phi)-\xi & \frac{1}{2} \phi \\
-6 \phi & -6+(4-2 \gamma) \phi+\psi-\xi
\end{array}\right) .
$$

Using the series representation of $\psi$, we further obtain, after a straightforward computation,

$$
\operatorname{Det}(\hat{E}(\theta)-\lambda(\theta) I)=\xi^{2}+a_{1} \xi+a_{2}
$$

where $a_{1}$ and $a_{2}$ are smooth functions of $\gamma$ and $\phi$ behaving for small $|\phi|$ as

$$
a_{1}=6+O(|\phi|), \quad a_{2}=\frac{1}{12}\left(1-\gamma+\gamma^{2}\right) \phi^{4}+O\left(|\phi|^{5}\right) .
$$

Thus, the eigenvalues behave for small $|\phi|$ as

$$
\lambda_{1}=e^{-i \gamma \theta}-\rho\left[c(\gamma) \phi^{4}+b_{1}(\gamma, \phi)\right], \quad \lambda_{2}=e^{-i \gamma \theta}-\rho\left[6+b_{2}(\gamma, \phi)\right],
$$

where $c(\gamma)=\frac{1}{72}\left(1-\gamma+\gamma^{2}\right)>0$ and $b_{1}$ and $b_{2}$ are smooth functions of $\gamma$ and $\phi$ in the range $\gamma \in[0,1],|\phi| \leqslant \kappa, \kappa$ small enough, with $b_{1}=O\left(|\phi|^{5}\right), b_{2}=O(|\phi|)$. Recalling that $\phi=i \theta+O\left(\theta^{2}\right)$, we obtain the asserted representation of the eigenvalues for small $|\phi|$.

The eigenvectors corresponding to the eigenvalues $\lambda_{1}$ and $\lambda_{2}$ are given respectively by

$$
V^{1}=\left(\begin{array}{c}
1 \\
i \theta
\end{array}\right)+O\left(\theta^{2}\right), \quad V^{2}=\left(\begin{array}{c}
\frac{1}{12} i \theta \\
1
\end{array}\right)+O\left(\theta^{2}\right)
$$

so that

$$
D(\theta)=\left(\begin{array}{cc}
1 & \frac{1}{12} i \theta \\
i \theta & 1
\end{array}\right)+O\left(\theta^{2}\right) .
$$

This proves the assertions concerning $D$, so the proof is complete.

LEMMA 3.3. There is a positive constant $\kappa$ such that $\hat{E}(\theta)$ allows the representation (3.10) in the range

$$
D_{\kappa}=\{(\theta, \gamma) \in[-\pi, \pi] \times[0,1]: \gamma(1-\gamma) \leqslant \kappa, \pi-|\theta| \leqslant \kappa\} .
$$

The eigenvalues of $\hat{E}(\theta)$ are smooth functions of $\gamma$ and $\theta$ in $D_{\kappa}$ satisfying

$$
\left|\lambda_{1,2}\right| \leqslant e^{-c \rho}, \quad(\gamma, \theta) \in D_{\kappa},
$$

where $c$ is a positive constant. Moreover, $|D(\theta)|$ and $\left|D^{-1}(\theta)\right|$ are bounded by a constant for all $(\theta, \gamma) \in D_{\kappa}$.

Proof. By a straightforward computation, the eigenvalues of $\hat{E}(\theta)$ are given for small $\gamma$ by

$$
\lambda_{1,2}=1-\left[2+e^{-i \theta}+\gamma a_{1}(\gamma, \theta)\right] \gamma+\left\{4+10 e^{-i \theta}-5 e^{-2 i \theta}+\gamma a_{2}(\gamma, \theta)\right\}^{1 / 2} \gamma,
$$

where $a_{1}$ and $a_{2}$ are smooth functions of $\gamma$ and $\theta$. By inspection, if $\kappa$ is small enough, the eigenvalues are smooth functions of $\gamma$ and $\theta$ in the range $\kappa \leqslant|\theta| \leqslant \pi-\kappa$ and satisfy $\left|\lambda_{1,2}\right| \leqslant e^{-c \gamma}$, where $c$ is a positive constant. Moreover, near the points 
$(\gamma, \theta)=(0, \pm \pi)$ the matrix $D$ in $(3.10)$ is given by

$$
D=\left(\begin{array}{cc}
1 & 1 \\
1-\sqrt{11} i & 1+\sqrt{11} i
\end{array}\right)+O(\gamma)+O\left(\left|e^{i \theta}+1\right|\right)
$$

which shows that $|D|$ and $\left|D^{-1}\right|$ are bounded in the range $\gamma \leqslant \kappa, \pi-|\theta| \leqslant \kappa, \kappa$ small enough.

The case where $\gamma$ is close to unity is handled similarly.

Lemma 3.4. Let $0<\kappa \leqslant \frac{1}{2}$. Then there is a positive constant $c$ depending only on $\kappa$ such that

$$
|\hat{E}(\theta)| \leqslant e^{-c \rho}, \quad \text { if } \kappa \leqslant|\theta| \leqslant \pi-\kappa \text { and } \gamma \in[0,1]
$$

and

$$
|\hat{E}(\theta)| \leqslant e^{-c} \text { if } \kappa \leqslant|\theta| \leqslant \pi \text { and } \max \{\gamma, 1-\gamma\} \geqslant \kappa .
$$

Proof. We need first some notation. For $V$ a vector, set $W=\hat{E}(\theta) V$ and define the functions $v, w$ and $z$ on $[0,1]$ as

$$
\begin{array}{r}
v(x)=V_{1}+V_{2}\left(x-\frac{1}{2}\right), \quad w(x)=W_{1}+W_{2}\left(x-\frac{1}{2}\right), \\
z(x)= \begin{cases}e^{-i \theta} v(x+1-\gamma), & 0 \leqslant x \leqslant \gamma, \\
v(x-\gamma), & \gamma<x \leqslant 1 .\end{cases}
\end{array}
$$

It follows from the definition of $\hat{E}(\theta)$ (see in particular (3.5) and (3.6)) that $w=\pi z$, where $\pi$ denotes the $L_{2}$-projection into the space of polynomials (with complex coefficients) of degree $\leqslant 1$ on $[0,1]$. Note that $\|z\|_{L_{2}[0,1]}=\|v\|_{L_{2}[0,1]}$ and that $z$ is a polynomial only if either $\rho=0$ or if $e^{-i \theta}=1$ and $V_{2}=0$.

Assume now that $\kappa \leqslant|\theta| \leqslant \pi-\kappa$. Then, since $\operatorname{Im}\left(e^{-i \theta}\right)$ is strictly nonzero, it is easy to verify that for any polynomial $p$ of degree $\leqslant 1$ one has the inequality

$$
\max \left\{\|z-p\|_{L_{\infty}[0, \gamma]},\|z-p\|_{L_{\infty}[1-\gamma, 1]}\right\} \geqslant C\|v\|_{L_{\infty}[0,1]}
$$

where $C$ is a positive constant depending only on $\kappa$. Setting $p=\pi z$, it follows easily that

$$
\|\pi z-z\|_{L_{2}[0,1]}^{2} \geqslant C \min \{\gamma, 1-\gamma\}\|v\|_{L_{2}[0,1]}^{2} \geqslant C \rho\|v\|_{L_{2}[0,1]}^{2} .
$$

By a similar reasoning, if $|\theta| \geqslant \kappa$ and $\rho \geqslant \kappa, \kappa>0$, there is a positive constant depending on $\kappa$ such that

$$
\|\pi z-z\|_{L_{2}[0,1]}^{2} \geqslant C\|v\|_{L_{2}[0,1]}^{2}
$$

Combining the last two inequalities we have

$$
\begin{aligned}
|\hat{E}(\theta) V|^{2} & =\|w\|_{L_{2}[0,1]}^{2}=\|z\|_{L_{2}[0,1]}^{2}-\|z-\pi z\|_{L_{2}[0,1]}^{2} \\
& \leqslant q\|v\|_{L_{2}[0,1]}^{2}=q|V|^{2},
\end{aligned}
$$

where $q=1-C(\kappa) \rho$ if $0<\kappa \leqslant|\theta| \leqslant \pi-\kappa$, and $q=q(\kappa)<1$ if $0<\kappa \leqslant|\theta| \leqslant \pi$ and $\rho \geqslant \kappa$. Since this is valid for any vector $V$, the proof is complete.

Remark. From the proof of Lemma 3.4 one might think that the inequality $|\hat{E}(\theta)| \leqslant e^{-c \rho}$ holds whenever $|\theta| \geqslant \kappa$, independently of $\gamma$. That this is not true is seen by choosing $v(x)=x-\frac{1}{2}$ and $\theta= \pm \pi$. Then

$$
\|\pi z-z\|_{L_{2}[0,1]}^{2} \leqslant\|v-z\|_{L_{2}[0,1]}^{2}=O\left(\rho^{3}\right),
$$


which shows that one can only have (and in fact has!) the estimate

$$
|\hat{E}(\theta)| \leqslant e^{-c \rho^{3}}, \quad|\theta| \geqslant \kappa, \gamma \in[0,1] .
$$

This is why Lemma 3.3 is needed (see below).

In the next lemma we combine the estimates of Lemmas 3.2 through 3.4.

LEMMA 3.5. Let $\tilde{E}(\theta)=e^{i \gamma \theta} \hat{E}(\theta)$. Then there are positive constants $C$ and $c$ such that for all $n \geqslant 1$ and $\theta \in[-\pi, \pi]$,

$$
\begin{aligned}
\left|\tilde{E}(\theta)^{n}\right| & \leqslant C e^{-c \rho n \theta^{4}}, \\
\left|\frac{d}{d \theta} \tilde{E}(\theta)^{n}\right| & \leqslant C\left(1+\rho n|\theta|^{3}\right) e^{-c \rho n \theta^{4}}+C(1+\rho n) e^{-c \rho n} .
\end{aligned}
$$

Proof. Choose $\kappa>0$ so that the assertions of Lemma 3.2 and Lemma 3.3 hold. Using the representation (3.10) together with Lemma 3.2 and Lemma 3.3, we then obtain

$$
\begin{aligned}
\left|\tilde{E}(\theta)^{n}\right| & \leqslant C \sum_{i=1}^{2}\left|\lambda_{i}(\theta)\right|^{n}, \\
\left|\frac{d}{d \theta} \tilde{E}(\theta)^{n}\right| & \leqslant C \sum_{i=1}^{2}\left(\left|\lambda_{i}(\theta)\right|^{n}+\left|\frac{d}{d \theta}\left[e^{i \gamma \theta} \lambda_{i}(\theta)\right]^{n}\right|\right),
\end{aligned}
$$

where $\lambda_{1,2}$ are the eigenvalues of $\hat{E}(\theta)$. From these estimates and from Lemma 3.2 and Lemma 3.3 the asserted estimates follow if $\kappa \leqslant|\theta| \leqslant \pi-\kappa$ or if $\pi-\kappa<|\theta| \leqslant \pi$ and $\max \{\gamma, 1-\gamma\} \geqslant \kappa$. In the remaining cases, we use Lemma 3.4 to obtain

$$
\begin{aligned}
\left|\frac{d}{d \theta} \tilde{E}(\theta)^{n}\right| & \leqslant n\left|\frac{d}{d \theta} \tilde{E}(\theta)\right||\tilde{E}(\theta)|^{n-1} \\
& =n\left|\frac{d}{d \theta}\left[e^{i \gamma \theta} A_{1}+e^{i(\gamma-1) \theta} A_{2}\right]\right||\tilde{E}(\theta)|^{n-1} \\
& \leqslant C \rho n|\tilde{E}(\theta)|^{n-1} \leqslant C^{\prime} \rho n e^{-c \rho n} .
\end{aligned}
$$

This completes the proof.

We are now ready to apply the Carlson-Beurling inequality for proving (3.8). Choosing $\alpha=\gamma n$ in Lemma 3.1 we conclude that

$$
\left\|U^{n}\right\|_{p, \mathbf{R}} \leqslant M_{p}\left(\tilde{E}^{n}\right)\left\|U^{0}\right\|_{p, \mathbf{R}}, \quad 1 \leqslant p \leqslant \infty,
$$

where $M_{p}\left(\tilde{E}^{n}\right)$ is estimated as

$$
M_{p}\left(\tilde{E}^{n}\right) \leqslant C\left\{\left\|\chi \tilde{E}^{n}\right\|_{L_{2}(\mathbf{R})}\left\|\frac{d}{d \theta} \chi \tilde{E}^{n}\right\|_{L_{2}(\mathbf{R})}\right\}^{1 / 2} .
$$

Using the estimates of Lemma 3.5 it follows that

$$
\left\|\chi \tilde{E}^{n}\right\|_{L_{2}(\mathbf{R})}^{2} \leqslant C \int_{0}^{3 \pi / 2} e^{-c \rho n \theta^{4}} d \theta \leqslant C_{1} \min \left\{1,(\rho n)^{-1 / 4}\right\}
$$

and

$$
\begin{aligned}
\left\|\frac{d}{d \theta} \chi \tilde{E}^{n}\right\|_{L_{2}(\mathbf{R})}^{2} & \leqslant C \int_{0}^{3 \pi / 2}\left\{\left[1+(\rho n)^{2}|\theta|^{6}\right] e^{-c \rho n \theta^{4}}+\left[1+(\rho n)^{2}\right] e^{-c \rho n}\right\} d \theta \\
& \leqslant C_{1}\left[1+(\rho n)^{1 / 4}\right] .
\end{aligned}
$$


Thus, $M_{p}\left(\tilde{E}^{n}\right)$ is bounded by a constant independent of $\rho$ and $n$. The estimate (3.8) now follows by combining (3.12) with the obvious estimates

$$
\left\|u_{h}^{-}\left(\cdot, t_{n}\right)\right\|_{p, \mathbf{R}} \leqslant C\left\|U^{n}\right\|_{p, \mathbf{R}}, \quad\left\|U^{0}\right\|_{p, \mathbf{R}} \leqslant C\|g\|_{p, \mathbf{R}} .
$$

It remains to prove (3.9). To this end, we will estimate the vector-valued function $W^{n}$ defined by

$$
\begin{gathered}
W^{n}=\left(W_{1}^{n}, W_{2}^{n}\right)^{T}, \quad W_{1}^{n}(x)=U_{1}^{n}(x)-U_{1}^{n}(x-h)-U_{2}^{n}(x), \\
W_{2}^{n}(x)=U_{2}^{n}(x)-U_{2}^{n}(x-h), \quad x \in \mathbf{R} .
\end{gathered}
$$

Here $U^{n}=\left(U_{1}^{n}, U_{2}^{n}\right)^{T}$ is defined by (3.7). Let us first show that it suffices to estimate $W^{n}$.

LEMma 3.6. There is a constant $C$ independent of $\gamma$ and $n$ such that

$$
\left\|\nu \cdot \beta\left(u_{h}^{+}-u_{h}^{-}\right)\right\|_{p, S_{n}} \leqslant C \rho\left\|W^{n}\right\|_{p, \mathbf{R}} .
$$

Proof. Let $v_{i}$ be a polynomial of degree $\leqslant 1$ on $\mathbf{R}^{2}$ such that $\left(u_{h}-v_{i}\right)_{\mid T_{n^{+}}}=0$ (see Figure 1). In the subdomain $\omega_{n}=\left\{(x, t): x \in \mathbf{R}, t_{n-1}<t<t_{n}\right\}$ we may interpret $w_{h}=u_{h}-v_{i}$ as the discontinuous Galerkin solution to the problem

$$
\frac{\partial u}{\partial t}+\gamma \frac{\partial u}{\partial x}=0 \quad \text { in } \omega_{n}, \quad u\left(\cdot, t_{n-1}\right)=\left(u_{h}^{-}-v_{i}\right)\left(\cdot, t_{n-1}\right)
$$

on the triangulation $\mathscr{C}_{n}^{h}=\left\{T \in \mathscr{C}^{h}: T \subset \omega_{n}\right\}$. Since $u_{h}-v_{i}$ vanishes on $T_{n i}^{+}$, we obtain by applying Lemma 2.1 and (3.3) that

$$
\begin{aligned}
\left\|\nu \cdot \beta\left(u_{h}^{+}-u_{h}^{-}\right)\right\|_{p,\left(\partial T_{n i}^{-}\right)_{-}} & =\left\|\nu \cdot \beta\left[\left(u_{h}-v_{i}\right)^{+}-\left(u_{h}-v_{i}\right)^{-}\right]\right\|_{p,\left(\partial T_{n i}^{-}\right)_{-}} \\
& \leqslant C \gamma\left\|u_{h}^{-}-v_{i}\right\|_{p,\left(\partial T_{n i}^{-}\right)_{-} \cap\left(\partial T_{n,-1}^{+}\right)_{+}} \\
& \leqslant C_{1} \gamma\left\|\left(u_{h}^{-}-v_{i}\right)\left(\cdot, t_{n-1}\right)\right\|_{L_{p}\left(x_{t-2}, x_{i-1}\right)}
\end{aligned}
$$

and similarly,

$$
\begin{aligned}
\left\|\nu \cdot \beta\left(u_{h}^{+}-u_{h}^{-}\right)\right\|_{p,\left(\partial T_{i i}^{-}\right)_{-}} & =\left\|\nu \cdot \beta\left[\left(u_{h}-v_{i-1}\right)^{+}-\left(u_{h}-v_{i-1}\right)^{-}\right]\right\|_{p,\left(\partial T_{n i}^{-}\right)-} \\
& \leqslant C(1-\gamma)\left\|\left(u_{h}^{-}-v_{i-1}\right)\left(\cdot, t_{n-1}\right)\right\|_{L_{p}\left(x_{i-1}, x_{i}\right)} .
\end{aligned}
$$

On the other hand, it follows from the definition of $W^{n}$ that $W^{n}$ vanishes on the interval $\left(x_{i-1}, x_{i}\right)$ if and only if $u_{h}^{-}\left(\cdot, t_{n-1}\right)$ is a polynomial of degree 1 on the interval $\left(x_{i-2}, x_{i}\right)$, i.e., if $u_{h}^{-}\left(x, t_{n-1}\right)=v_{i}(x)=v_{i-1}(x)$ for $x \in\left(x_{i-2}, x_{i}\right)$. From this it follows easily that

$$
\left\|u_{h}^{-}\left(\cdot, t_{n}\right)-v_{i}\right\|_{L_{p}\left(x_{i-2}, x_{i-1}\right)} \leqslant C\left\|W^{n}\right\|_{L_{p}\left(x_{i-1}, x_{i}\right)}
$$

and

$$
\left\|u_{h}^{-}\left(\cdot, t_{n}\right)-v_{i-1}\right\|_{L_{p}\left(x_{i-1}, x_{i}\right)} \leqslant C\left\|W^{n}\right\|_{L_{p}\left(x_{i}, 1, x_{i}\right)} .
$$

Combining these inequalities we obtain

$$
\left\|\nu \cdot \beta\left(u_{h}^{+}-u_{h}^{-}\right)\right\|_{p,\left(\partial T_{n l}^{+}\right)_{-}} \leqslant C \rho\left\|W^{n}\right\|_{L_{p}\left(x_{i-1}, x_{i}\right)} .
$$

This proves the assertion for $p=\infty$, and for $p<\infty$ it remains only to sum over $i$. 
Let us now complete the proof by estimating $\left\|W^{n}\right\|_{p}$. Note first that the Fourier transform of $W^{n}$ is given by

$$
\hat{W}^{n}(\xi)=B(h \xi) \hat{U}^{n}(\xi)=\left(B \hat{E}^{n}\right)(h \xi) \hat{U}^{0}(\xi),
$$

where

$$
B(\theta)=\left(\begin{array}{cc}
1-e^{-i \theta} & -1 \\
0 & 1-e^{-i \theta}
\end{array}\right) .
$$

Assume first that $|\theta|$ is small enough. Then using the diagonalization (3.10) of $\hat{E}(\theta)$ and recalling (3.11) we obtain after a simple computation

$$
B \hat{E}^{n}=\lambda_{1}^{n} H_{1}+\lambda_{2}^{n} H_{2}
$$

where $H_{1}=O\left(\theta^{2}\right)$. Therefore, using Lemma 3.2, we conclude that for $|\theta|<\kappa, \kappa$ small enough,

$$
\begin{aligned}
\left|B \tilde{E}^{n}(\theta)\right| & \leqslant C\left(\theta^{2} e^{-c \rho n \theta^{4}}+e^{-c \rho n}\right), \\
\left|\frac{d}{d \theta} B \tilde{E}^{n}(\theta)\right| & \leqslant C\left[\left(|\theta|+\rho n|\theta|^{5}\right) e^{-c \rho n \theta^{4}}+(1+\rho n) e^{-c \rho n}\right] .
\end{aligned}
$$

Using a similar argument as in the proof of Lemma 3.5, we conclude that these estimates remain valid also for $\kappa \leqslant|\theta| \leqslant \pi$. We can now apply the Carlson-Beurling inequality to obtain

$$
\|W\|_{p, \mathbf{R}} \leqslant M_{p}\left(B \tilde{E}^{n}\right)\left\|U^{0}\right\|_{p, \mathbf{R}},
$$

where $M_{p}\left(B \tilde{E}^{n}\right)$ is estimated as in Lemma 3.1. An easy computation shows that

$$
M_{p}\left(B \tilde{E}^{n}\right) \leqslant C(\rho n)^{-1 / 2} \text {. }
$$

Combining the last two estimates with that given in Lemma 3.6 we now end up with (3.9), and the proof of Theorem 3.1 is complete.

We conclude this section by stating a stability estimate for the discontinuous Galerkin method applied to the problem

$$
\begin{gathered}
\frac{\partial u}{\partial t}+\gamma \frac{\partial u}{\partial x}=0, \quad(x, t) \in \Omega_{n}, \\
u=g, \quad(x, t) \in\left(\partial \Omega_{n}\right)_{-},
\end{gathered}
$$

where $\gamma \in[0,1]$ and $\Omega_{n}, n \geqslant 1$, is the triangle

$$
\Omega_{n}=\left\{(x, t) ; 0<t<t_{n}=n h, 0<x<t\right\} .
$$

Let the triangulation $\mathscr{C}^{h}$ of $\Omega_{n}$ be defined as the restriction of the above triangulation of the half-plane to $\Omega_{n}$, let $k=1$ and let $u_{h}$ be defined according to (1.2) on each $T \in \mathscr{C}^{h}$. Then we have in analogy with (3.8) the following stability estimate.

THEOREM 3.2. There is a constant $C$ such that for all $n \geqslant 1$ and $1 \leqslant p \leqslant \infty$,

$$
\left\|u_{h}^{-}\left(\cdot, t_{n}\right)\right\|_{L_{p}\left(0, t_{n}\right)}+\left\|u_{h}\right\|_{p, \Omega_{n}} \leqslant C\|\nu \cdot \beta g\|_{p .\left(\partial \Omega_{n}\right)_{-}} .
$$

The proof is based on the following localization result.

LEMMA 3.7. Write the solutions of (3.7) as

$$
U^{n}(x)=\sum_{j=-\infty}^{+\infty} B_{n j} U^{0}(x-j h), \quad x \in \mathbf{R} .
$$


Then the matrices $B_{n j}$ satisfy

$$
\left|B_{n j}\right| \leqslant C \min \left\{1,(\rho n)^{-1 / 4},\left[1+(\rho n)^{1 / 4}\right](j-\gamma n)^{-2}\right\} .
$$

Proof. Using the Fourier transform $\hat{E}(\theta)$ of $G_{h}$ we have

$$
B_{n j}=\frac{1}{2 \pi} \int_{-\pi}^{\pi} \hat{E}(\theta)^{n} e^{i j \theta} d \theta
$$

By Lemma 3.1,

$$
\left|B_{n j}\right| \leqslant \frac{C}{2 \pi} \int_{-\pi}^{\pi} e^{-c \rho n \theta^{4}} d \theta \leqslant C \min \left\{1,(\rho n)^{-1 / 4}\right\} .
$$

On the other hand, integrating by parts and using the periodicity of $\hat{E}(\theta)$, we have

$$
\begin{aligned}
B_{n j} & =\frac{1}{2 \pi} \int_{-\pi}^{\pi} \tilde{E}(\theta)^{n} e^{i(j-n \gamma) \theta} d \theta \\
& =-\frac{1}{2 \pi(j-n \gamma)^{2}} \int_{-\pi}^{\pi} \frac{d^{2}}{d \theta^{2}}\left[\tilde{E}(\theta)^{n}\right] e^{i(j-n \gamma) \theta} d \theta .
\end{aligned}
$$

From the argument used in the proof of Lemma 3.5, it is easy to see that

$$
\begin{aligned}
\left|\frac{d^{2}}{d \theta^{2}}\left[\tilde{E}(\theta)^{n}\right]\right| \leqslant C\left[\left(1+\rho n \theta^{2}+(\rho n)^{2} \theta^{6}\right) e^{-c \rho n \theta^{4}}\right. & \\
& \left.+\left(1+\rho n+(\rho n)^{2} e^{-c \rho n}\right)\right], \quad 0 \leqslant|\theta| \leqslant \pi .
\end{aligned}
$$

Using this estimate we obtain

$$
\left|B_{n j}\right| \leqslant C\left[1+(\rho n)^{1 / 4}\right](j-\gamma n)^{-2},
$$

and so the assertion is proved.

Remark. Localization estimates similar to those given by Lemma 3.7 are presented, e.g., in [3], [5] for scalar difference schemes. When compared with these estimates, Lemma 3.7 indicates that on a uniform mesh, the discontinuous Galerkin scheme with $k=1$ behaves like a finite difference scheme which is accurate of order three and dissipative of order four.

Let us now prove Theorem 3.2. For $1 \leqslant m \leqslant n$, define

$$
g_{m}(x, t)= \begin{cases}g(x, t), & \text { if }(x, t) \in\left(\partial \Omega_{n}\right)_{-} \text {and } t_{m-1}<t<t_{m}, \\ 0, & \text { elsewhere on }\left(\partial \Omega_{n}\right)_{-} .\end{cases}
$$

Then

$$
u_{h}^{-}\left(x, t_{n}\right)=\sum_{m=1}^{n} u_{h m}^{-}\left(x, t_{n}\right), \quad 0<x<t_{n},
$$

where $u_{h m}$ denotes the approximate solution of (3.13) with $g$ replaced by $g_{m}$. Now let $m$ be given $1 \leqslant m \leqslant n$, and define for $m \leqslant r \leqslant n$ the vector-valued function $U^{r-m}$ on $\mathbf{R}$ by

$$
U^{r-m}(x)=\left[u_{h m}^{-}\left(x_{i+1 / 2}, t_{r}\right), h \frac{\partial u_{h m}}{\partial x}\left(x_{i+1 / 2}, t_{r}\right)\right]^{T}, \quad x_{i} \leqslant x<x_{i+1},
$$


where we set $u_{h m}=0$ outside $\Omega_{n}$. Then $U^{r-m}=E^{r-m} U^{0}$, where $E$ is as in (3.7) and $U^{0}$ is nonvanishing on the intervals $\left(x_{0}, x_{1}\right)$ and $\left(x_{m-1}, x_{m}\right)$ only. Moreover, applying Lemma 2.1, we have the estimates

$$
\left\|U^{0}\right\|_{L_{\infty}\left(x_{0}, x_{1}\right)} \leqslant C \gamma\|g(0, \cdot)\|_{L_{\infty}\left(t_{m-1}, t_{m}\right)} \leqslant C \gamma\|g\|_{\infty,\left(\partial \Omega_{n}\right)_{-}},
$$

and, similarly,

$$
\left\|U^{0}\right\|_{L_{\infty}\left(x_{m-1}, x_{m}\right)} \leqslant C(1-\gamma)\|g\|_{\infty,\left(\partial \Omega_{\left.n_{1}\right)_{-}} .\right.}
$$

On the other hand, by Lemma 3.7 we have

$$
\begin{aligned}
q\left|U^{n-m}\left(x_{i+1 / 2}\right)\right| \leqslant & q_{1}(n-m, i)\left|U^{0}\left(x_{1 / 2}\right)\right| \\
& +q_{2}(n-m, i)\left|U^{0}\left(x_{m-1 / 2}\right)\right|, \quad 0 \leqslant i \leqslant n-1,
\end{aligned}
$$

where $q_{1}$ and $q_{2}$ satisfy

$$
\begin{aligned}
& q_{1}(r, i) \leqslant C \min \left\{1,(\rho r)^{-1 / 4},\left[1+(\rho r)^{1 / 4}\right](i-\gamma r)^{-2}\right\} \\
& q_{2}(r, i) \leqslant C \min \left\{1,(\rho r)^{-1 / 4},\left[1+(\rho r)^{1 / 4}\right](m-1-i-\gamma r)^{-2}\right\} .
\end{aligned}
$$

Combining the above inequalities and summing over $m$ we now obtain

$$
\begin{aligned}
& \left\|u_{h}^{-}\left(\cdot, t_{n}\right)\right\|_{L_{\infty}\left(x_{i}, x_{i+1}\right)} \\
& \quad \leqslant C\|g\|_{\infty,\left(\partial \Omega_{n}\right)_{-}}\left\{\gamma \sum_{r=0}^{\infty} q_{1}(r, i)+(1-\gamma) \sum_{r=0}^{\infty} q_{2}(r, i)\right\} .
\end{aligned}
$$

Using the above estimate for $q_{1}(r, i)$ we may estimate the first sum on the right side of (3.14) as

$$
\begin{aligned}
\gamma \sum_{m=0}^{\infty} q_{1}(r, i) \leqslant & C \gamma \sum_{r:|i-\gamma r| \leqslant d} \min \left\{1,(\rho r)^{-1 / 4}\right\} \\
& +C \gamma \sum_{r:|i-\gamma r|>d}\left[1+(\rho r)^{1 / 4}\right](i-\gamma r)^{-2}
\end{aligned}
$$

Choosing here

$$
d=\max \left\{1,(\rho i / \gamma)^{1 / 4}\right\},
$$

it is easy to see that both sums on the right side are bounded by an absolute constant. Upon estimating the second sum on the right side of (3.14) in a similar manner we now obtain

$$
\left\|u_{h}^{-}\left(\cdot, t_{n}\right)\right\|_{L_{\infty}\left(0, x_{n}\right)} \leqslant C\|g\|_{\infty,\left(\partial \Omega_{n}\right)_{-}, \quad n \geqslant 1 .}
$$

Combining this estimate with the local estimates given in Lemma 2.1, the assertion follows in the case $p=\infty$. The case $p=1$ can be handled in a similar manner, and finally the remaining cases can be treated by interpolation [1]. We omit these details.

Remark 3.1. In the above analysis we have confined ourselves to the case $k=1$. The case $k=0$ is more elementary, and one can easily verify that the estimates stated in Theorems 3.1 and 3.2 are valid also in this case. Note that if $k=0,(3.8)$ merely states the well-known $L_{p}$-stability of the upwind finite difference scheme, cf. [4]. So far we have not been able to carry out the full $L_{p}$-stability analysis of the scheme (3.7) when $k \geqslant 2$. 
Remark 3.2. Using the stability estimate (3.8) one can perform an ordinary finite difference error analysis of the discontinuous Galerkin method when applied to problem (3.1). As an example, let us estimate the error of $u_{h}\left(\cdot, t_{n}\right)$ for some choices of initial data assuming that $k=1$ and $n \leqslant C h^{-1}$.

Case 1. Let us first seek a scheme of maximal order of accuracy by choosing the initial data as

$$
U^{0}(x)=\left(g(x), c_{1} \Delta_{h} g(x)+c_{2} \Delta_{h}^{2} g(x)\right)
$$

where $\Delta_{h} g(x)=g(x)-g(x-h)$ and $c_{1}$ and $c_{2}$ are constants to be defined shortly. We have for the discrete solution $U$

$$
\hat{U}^{n}(\xi)=[\hat{E}(h \xi)]^{n} \hat{U}^{0}(\xi) .
$$

Correspondingly, we have for the exact solution, defining

$$
W^{n}(x)=\left(u\left(x, t_{n}\right), c_{1} \Delta_{h} u\left(x, t_{n}\right)+c_{2} \Delta_{h}^{2} u\left(x, t_{n}\right)\right),
$$

that

$$
\hat{W}^{n}(\xi)=e^{-i \gamma n h \xi} \hat{W}^{0}(\xi)=e^{-i \gamma n h \xi} \hat{U}^{0}(\xi) .
$$

Let us now choose the constants $c_{1}$ and $c_{2}$ so that $\hat{U}^{0}(\xi)=\hat{g}(\xi)\left[V(\xi)+O\left(|h \xi|^{3}\right)\right]$, where $V(\xi)=\left(1, V_{2}(\xi)\right)$ is the eigenvector of $\hat{E}(h \xi)$ corresponding to the eigenvalue $\lambda_{1}(\xi)=e^{-i \gamma h \xi}+O\left((h \xi)^{4}\right)$. From the proof of Lemma 3.2 we obtain

$$
c_{1}=1, \quad c_{2}=\frac{1}{3}(2-\gamma) \text {. }
$$

With this choice we have, for $n h \leqslant C$,

$$
\begin{aligned}
\hat{W}^{n}(\xi)-\hat{U}^{n}(\xi) & =e^{-i \gamma n h \xi} \hat{W}^{0}(\xi)-\lambda_{1}(\xi)^{n} V(\xi)+O\left(|h \xi|^{3}\right) \\
& =\left[e^{-i \gamma n h \xi}-\lambda_{1}(\xi)^{n}\right] \hat{W}^{0}(\xi)+O\left(|h \xi|^{3}\right) \\
& =O\left(h^{3} \xi^{4}\right) \hat{W}^{0}(\xi)+O\left(|h \xi|^{3}\right) .
\end{aligned}
$$

This leads to the error estimate

$$
\left|u\left(x_{i+1 / 2}, t_{n}\right)-u_{h}^{-}\left(x_{i+1 / 2}, t_{n}\right)\right| \leqslant C h^{3}\|g\|_{W^{4 . \infty}(\mathbf{R})},
$$

so with a proper choice of initial data, the scheme is accurate of order three. Obviously, the high accuracy can only be achieved at discrete points; if the error is measured in the norm $\|\cdot\|_{p, \mathbf{R}}$, a finite difference analysis only gives

$$
\left\|\left(u-u_{h}^{-}\right)\left(\cdot, t_{n}\right)\right\|_{p, \mathbf{R}} \leqslant C h\|g\|_{W^{2, p}(\mathbf{R})},
$$

which is an estimate typical for first-order schemes. We see below in Section 4 that the latter estimate can be improved by using the improved stability estimate (3.9).

Case 2. Let the initial data be chosen as

$$
U^{0}(x)=\left(\pi_{h} g(x), h \frac{\partial}{\partial x}\left(\pi_{h} g\right)(x)\right),
$$

which is the choice made by the usual discontinuous Galerkin scheme. Then

$$
\left\|U^{0}-W^{0}\right\|_{p, \mathbf{R}} \leqslant C h^{2}\|g\|_{W^{3, p}(\mathbf{R})},
$$

which gives

$$
\left|\left(u-u_{h}^{-}\right)\left(x_{i+1 / 2}, t_{n}\right)\right| \leqslant C h^{2}\|g\|_{W^{3 . \infty}(\mathbf{R})} .
$$


To sum up, we have for $k=1$ and $n \leqslant C h^{-1}$,

$$
\left|\left(u-u_{h}^{-}\right)\left(x_{i+1 / 2}, t_{n}\right)\right| \leqslant C h^{s}\|g\|_{W^{s+1 . \infty}(\mathbf{R})},
$$

where $s=3$ with a special choice of initial data and $s=2$ with the usual choice of data. Moreover, for (essentially) all choices of initial data we have

$$
\left\|\left(u-u_{h}^{-}\right)\left(\cdot, t_{n}\right)\right\|_{p, \mathbf{R}} \leqslant C h\|g\|_{w^{2, p}(\mathbf{R}),} \quad 1 \leqslant p \leqslant \infty .
$$

4. $L_{p}$-Error Estimates. We now apply the results of the previous section to derive $L_{p}$-error estimates for the scheme (1.2) in the case where $\mathscr{C}^{h}$ is a uniform or piecewise-uniform triangulation of $\Omega$ and either $k=0$ or $k=1$. In the case of a uniform triangulation we will only need the estimates of Theorem 3.1, whereas if the triangulation is only piecewise uniform, also Theorem 3.2 will be required (in the case $p=\infty$ ). The results below are thus valid for any value of $k$ for which the estimates of Theorem 3.1 and 3.2 can be proved.

We will need stability estimates analogous to (3.8) and (3.9) for the discontinuous Galerkin method applied to the problem

$$
\begin{cases}u_{\beta}+a u=f & \text { in } \Omega, \\ u=g & \text { on } \partial \Omega_{-},\end{cases}
$$

where $a \in L_{\infty}(\Omega)$. To generalize the situation stepwise, let us first assume that $\Omega$ is still the half-plane, with $u_{\beta}=\partial u / \partial t+\gamma \partial u / \partial x$ and $\gamma \in[0,1]$ as above and with the triangulation $\mathscr{C}^{h}$ defined as in the previous section. For each $h$ we further use the notation

$$
\begin{aligned}
& \omega_{n}=\left\{(x, t): x \in \mathbf{R}, t_{n-1}<t<t_{n}\right\} \\
& \Omega_{n}=\left\{(x, t): x \in \mathbf{R}, 0<t<t_{n}\right\}, \\
& \Gamma_{n}=\bigcup_{m=1}^{n} S_{m},
\end{aligned}
$$

where $t_{n}=n h$ and $S_{m}$ is defined in Section 3. We now prove

THEOREM 4.1. Let $\mathscr{C}^{h}$ be as above and let $u_{h}$ be the approximate solution of (4.1) defined by (2.4) with either $k=0$ or $k=1$ and with $h\|a\|_{\infty, \Omega}$ sufficiently small. Then we have for all $n \geqslant 1$ and $1 \leqslant p \leqslant \infty$ the estimates

$$
\begin{aligned}
& \left\|u_{h}^{-}\left(\cdot, t_{n}\right)\right\|_{p, \mathbf{R}}+\left\|u_{h}\right\|_{p, \Omega_{n}} \\
& \quad \leqslant C M q^{n}(h n)^{1-1 / p}\left(\|f\|_{p, \Omega_{n}}+\|g\|_{p, \mathbf{R}}\right)
\end{aligned}
$$

and

$$
\begin{aligned}
\| \nu \cdot & \beta\left(u_{h}^{+}-u_{h}^{-}\right) \|_{p, \Gamma_{n}} \\
& \leqslant C M^{2} q^{n}\left[h^{1-1 / p} n^{1 / 2}(h n)^{1-1 / p}\|f\|_{p, \Omega_{n}}+n^{\max \{0,1 / p-1 / 2\}}\|g\|_{p, \mathbf{R}}\right],
\end{aligned}
$$

where $M=1+\|a\|_{\infty, \Omega_{n}}, q=1+C M h$ and $C$ is an absolute constant.

Proof. Let us derive first some local estimates for $u_{h}$ on $\omega_{n}$. Applying repeatedly Lemma 2.1 to the triangles $T_{n, i-1}^{+}, T_{n i}^{+}$and $T_{n i}^{-}$(see Figure 1), we see that

$$
\begin{aligned}
& \left\|u_{h}\right\|_{p, K_{i}}+h^{1 / p}\left\|u_{h}^{-}\left(\cdot, t_{n}\right)\right\|_{L_{p}\left(x_{i-1}, x_{i}\right)}+h^{1 / p}\left\|\nu \cdot \beta\left(u_{h}^{+}-u_{h}^{-}\right)\right\|_{p,\left(T_{n i}^{-}\right)} \\
& \quad \leqslant C h\|f\|_{p, K_{i}}+C h^{1 / p}\left\|u_{h}^{-}\left(\cdot, t_{n-1}\right)\right\|_{L_{p}\left(x_{i-2}, x_{i}\right)},
\end{aligned}
$$


where $K_{i}=T_{n, i-1}^{+} \cup T_{n i}^{+} \cup T_{n i}^{-}$. For $p=\infty$ this implies immediately that

$$
\begin{aligned}
& \left\|u_{h}\right\|_{p, \omega_{n}}+h^{1 / p}\left\|u_{h}^{-}\left(\cdot, t_{n}\right)\right\|_{p, \mathbf{R}}+h^{1 / p}\left\|_{\nu} \cdot \beta\left(u_{h}^{+}-u_{h}^{-}\right)\right\|_{p, S_{n}} \\
& \quad \leqslant C h\|f\|_{\omega_{n}}+C h^{1 / p}\left\|u_{h}^{-}\left(\cdot, t_{n-1}\right)\right\|_{p, \mathbf{R}},
\end{aligned}
$$

and for $1 \leqslant p<\infty$ the same estimate readily follows by summation over $i$.

It is easy to sharpen the estimate for $u_{h}^{-}\left(\cdot, t_{n}\right)$ in (4.3) by writing

$$
u_{h}^{-}\left(\cdot, t_{n}\right)=G_{h} u_{h}^{-}\left(\cdot, t_{n-1}\right)+v_{n}\left(\cdot, t_{n}\right), \quad n \geqslant 1,
$$

where $G_{h}$ is defined by (3.4) and (3.5). By linearity, $v_{n}$ is the discontinuous Galerkin solution on $\omega_{n}$ to the problem

$$
\left\{\begin{array}{l}
\frac{\partial w}{\partial t}+\gamma \frac{\partial w}{\partial x}=f_{n}, \quad(x, t) \in \omega_{n} \\
u\left(\cdot, t_{n-1}\right)=0
\end{array}\right.
$$

where $f_{n}=f-a u_{h}$. Thus, by (4.3), $v_{n}$ satisfies

$$
\begin{aligned}
& \left\|v_{n}\left(\cdot, t_{n}\right)\right\|_{p, \mathbf{R}} \leqslant C h^{1-1 / p}\left\|f_{n}\right\|_{p, \omega_{n}} \\
& \quad \leqslant C_{1} M\left(h\left\|u_{h}^{-}\left(\cdot, t_{n-1}\right)\right\|_{p, \mathbf{R}}+h^{1-1 / p}\|f\|_{p, \omega_{n}}\right) .
\end{aligned}
$$

Upon solving for $u_{h}$ in (4.4), we get

$$
u_{h}^{-}\left(\cdot, t_{n}\right)=\sum_{j=0}^{n} G_{h}^{n-1} w_{j}, \quad w_{j}=v_{j}\left(\cdot, t_{j}\right), \quad j \geqslant 1, w_{0}=g .
$$

Applying here (4.5) and recalling that, by Theorem 3.1,

$$
\left\|G_{h}^{n} g\right\|_{p, \mathbf{R}} \leqslant C\|g\|_{p, \mathbf{R}}, \quad g \in L_{p}(\mathbf{R}), n \geqslant 1,1 \leqslant p \leqslant \infty,
$$

we see that for any $n \geqslant 1$,

$$
\left\|u_{h}^{-}\left(\cdot, t_{n}\right)\right\|_{p, \mathbf{R}} \leqslant C M h \sum_{j=0}^{n-1}\left\|u_{h}^{-}\left(\cdot, t_{n}\right)\right\|_{p, \mathbf{R}}+C M(h n)^{1-1 / p}\|f\|_{p, \Omega_{n}} .
$$

The iteration of this inequality now gives

$$
\left\|u_{h}^{-}\left(\cdot, t_{n}\right)\right\|_{p, \mathbf{R}} \leqslant C(1+C M h)^{n}\left[M(h n)^{1-1 / p}\|f\|_{p, \Omega_{n}}+\|g\|_{p, \mathbf{R}}\right],
$$

which proves the first estimate in the theorem.

In the remaining part of the proof we use the splitting

$$
u_{h}=\sum_{m=0}^{n} u_{h m}, \quad(x, t) \in \Omega_{n},
$$

where for $m \geqslant 1, u_{h m}$ denotes the discontinuous Galerkin solution to the problem

$$
\begin{gathered}
\frac{\partial w}{\partial t}+\frac{\partial w}{\partial x}= \begin{cases}f-a u_{h}, & (x, t) \in \omega_{m}, \\
0, & \text { elsewhere on } \Omega,\end{cases} \\
u(\cdot, 0)=0,
\end{gathered}
$$

and $u_{h 0}$ is the discontinuous Galerkin solution to $\partial w / \partial t+\gamma \partial w / \partial x=0$ in $\Omega$, $w(\cdot, 0)=g$. Since $u_{h m}$ obviously vanishes for $t<t_{m-1}$, we have by (4.3) that for $m \geqslant 1$

$$
\left\|\nu \cdot \beta\left(u_{h m}^{+}-u_{h m}^{-}\right)\right\|_{p, S_{m}}+\left\|u_{h m}^{-}\left(\cdot, t_{n}\right)\right\|_{p, \mathbf{R}} \leqslant C h^{1-1 / p}\left\|f-a u_{h}\right\|_{p, \omega_{m}} .
$$


Now Theorem 3.1 implies that for $j>m \geqslant 1$,

$$
\left\|\nu \cdot \beta\left(u_{h m}^{+}-u_{h m}^{-}\right)\right\|_{p, S} \leqslant C(j-m)^{-1 / 2} h^{1-1 / p}\left\|f-a u_{h}\right\|_{p, \omega_{m}},
$$

and

$$
\left\|\nu \cdot \beta\left(u_{h 0}^{+}-u_{h 0}^{-}\right)\right\|_{p, S_{j}} \leqslant C j^{-1 / 2}\|g\|_{p, \mathbf{R}} .
$$

Combining this with (4.7) and summing over $m$, recalling (4.6), we have

$$
\left\|\nu \cdot \beta\left(u_{h}^{+}-u_{h}^{-}\right)\right\|_{\infty, \Gamma_{n}} \leqslant C\left[h n^{1 / 2}\left\|f-a u_{h}\right\|_{\infty, \Omega_{n}}+\|g\|_{\infty, \mathbf{R}}\right],
$$

and

$$
\begin{aligned}
& \sum_{j=1}^{n}\left\|\nu \cdot \beta\left(u_{h}^{+}-u_{h}^{-}\right)\right\|_{1, S_{j}}=\left\|\nu \cdot \beta\left(u_{h}^{+}-u_{h}^{-}\right)\right\|_{1, \Gamma_{n}} \\
& \quad \leqslant C n^{1 / 2}\left[\left\|f-a u_{h}\right\|_{\Omega_{n}}+\|g\|_{1, \mathbf{R}}\right] .
\end{aligned}
$$

When combined with the estimate already proved for $\left\|u_{h}\right\|_{p, \Omega_{n}}$, these inequalities prove the second estimate of the theorem in the cases $p=\infty$ and $p=1$. The remaining values of $p$ can be treated similarly, and so the proof is complete.

Let us now return to the original situation of Eq. (1.1) where $\Omega$ is a bounded convex polygonal domain. We assume first that $\Omega$ allows a uniform triangulation, which obviously is possible only in specific cases. To simplify the notation, let us reduce the situation to that of (4.1) by introducing the affine mapping $F: \mathbf{R}^{2} \rightarrow \mathbf{R}^{2}$ with the following properties: (i) if $\tilde{\beta}=F(\beta)$ then $\tilde{\beta}_{2} / \tilde{\beta}_{1} \in[0,1]$, (ii) if $T \in \mathscr{C}^{h}$ then for some $n, 0 \leqslant n \leqslant C h^{-1} \operatorname{diam} \Omega$ and for some $i, F(T)$ has two of its vertices at the points $(i h, n h)$ and $((i-1) h,(n-1) h)$ and the third vertex either at $((i-1) h, n h)$ or at $(i h,(n-1) h)$. It is obvious that such a mapping exists and is nonsingular. Moreover, if we write $\tilde{v}(F(x))=v(x), x \in \Omega$ and $\tilde{\Omega}=F(\Omega)$, it is easy to see that $\tilde{u}_{h}$ is the discontinuous Galerkin solution of the transformed problem

$$
\begin{aligned}
\tilde{\beta} \cdot \nabla \tilde{u}+\tilde{a} \tilde{u} & =\tilde{f} & & \text { in } \tilde{\Omega}, \\
\tilde{u} & =\tilde{g} & & \text { on } \partial \tilde{\Omega}{ }_{-},
\end{aligned}
$$

on the triangulation $\left\{\tilde{T}=F(T): T \in \mathscr{C}^{h}\right\}$. Note also that $C^{-1} \leqslant|\tilde{\beta}| \leqslant C$ and that the error estimates to be proved below are invariant under the transformation $F$. Thus, we may as well consider the transformed problem. Below we suppress the tildes for simplicity, i.e., we write $\Omega$ instead of $\tilde{\Omega}, u_{h}$ instead of $\tilde{u}_{h}$, etc. Moreover, we use the coordinates $(x, t)$ instead of $\left(x_{1}, x_{2}\right)$ and denote by $\mathscr{M}^{h}$ the uniform triangulation of the half-plane $\{(x, t): x \in \mathbf{R}, t>0\}$ such that $\mathscr{C}^{h}=\left\{T \in \mathscr{M}^{h}\right.$ : $T \subset \Omega\}$, i.e., $\mathscr{M}_{h}$ coincides with the triangulation $\mathscr{C}^{h}$ referred to in Theorem 4.1. We also use the notation

$$
D_{j}=\left\{x \in \mathbf{R}:\left(x, t_{j}\right) \in \Omega\right\} .
$$

We can now prove

THEOREM 4.2. Let the domain $\Omega$ and the triangulation $\mathscr{C}^{h}=\mathscr{M}_{\mid \Omega}^{h}$ be as above, let $u$ be the solution of (1.1) and let $u_{h}$ be defined by (1.2) with either $k=0$ or $k=1$. Assume further that $h\|a\|_{\infty, \Omega}$ is small enough and that $h^{1 / 2}\|a\|_{\infty, \Omega} \leqslant C$. Then we have the error estimates

$$
\left\|u-u_{h}\right\|_{p, \Omega} \leqslant C M^{2} h^{k+1 / 2}|u|_{W^{k+1, p}(\Omega)}, \quad 1 \leqslant p \leqslant \infty,
$$


and

$$
\begin{aligned}
& \max _{j: D_{j} \neq \phi}\left\|\left(u-u_{h}\right)\left(\cdot, t_{j}\right)\right\|_{p, D_{j}} \\
& \quad \leqslant C M^{2} \begin{cases}h^{k+1 / 2}|u|_{W^{k+1 . p}(\Omega)}, & p \geqslant 2, \\
h^{k+1-1 / p}|u|_{W^{k+1 . p}(\Omega)}, & 1 \leqslant p \leqslant 2\end{cases}
\end{aligned}
$$

where $M=1+\|a\|_{\infty, \Omega}$ and $C$ is a constant depending only on $\operatorname{diam}(\Omega)$.

Proof. Let $n$ be sufficiently large so that $\Omega \subset \Omega_{n}=\{(x, t): x \in \mathbf{R}, 0<t<n h\}$ and let $\varphi \in L_{1}\left(\Omega_{n}\right)$ be such that $\varphi$ vanishes outside $\Omega$. Define the function $\phi_{h}$ on $\Omega_{n}$ so that $\phi_{\left.h\right|_{T}} \in P_{k}(T), T \in \mathscr{M}_{h}$, and

$$
\begin{gathered}
\int_{T} v\left(-\phi_{h \beta}+a \phi_{h}\right) d x d t+\int_{\partial T_{+}} v^{-}\left(\phi_{h}^{-}-\phi_{h}^{+}\right) \nu \cdot \beta d s \\
=\int_{T} \varphi v d x d t, \quad T \in \mathscr{M}_{h}, T \subset \Omega_{n},
\end{gathered}
$$

where $\phi_{h}^{+}=0$ on $\left(\partial \Omega_{n}\right)_{+}$and $a=0$ outside $\Omega$. Via the simple coordinate transformation $t \rightarrow n h-t, \phi_{h}$ becomes the discontinuous Galerkin solution of the problem (4.1) with $\Omega=\Omega_{n}$ and $f(x, t)=\varphi(x, n h-t)$ and $g=0$. Thus, by Theorem 4.1 and since $n \leqslant C \operatorname{diam}(\Omega) h^{-1}$,

$$
\begin{gathered}
\left\|\phi_{h}\right\|_{p, \Omega_{n}} \leqslant C M\left\|_{\varphi}\right\|_{p, \Omega}, \\
\left\|\nu \cdot \beta\left(\phi_{h}^{+}-\phi_{h}^{-}\right)\right\|_{p, \Gamma_{n}} \leqslant C M^{2} h^{1 / 2-1 / p}\|\phi\|_{p, \Omega}, \quad \varphi_{\mid \Omega} \in L_{p}(\Omega) .
\end{gathered}
$$

Let us now sum over $T \subset \Omega$ in (4.8) to obtain

$$
\mathscr{B}\left(v, \phi_{h}\right)=(\varphi, v), \quad v \in V_{h},
$$

where $V_{h}$ is as in (2.3) and $\mathscr{B}$ is defined by (2.5) or equivalently, by (2.6). Choosing here $v=u_{h}-\tilde{u}$, where $\tilde{u} \in V_{h}$ is an interpolant of $u$ to be defined below and recalling (2.7), we obtain

$$
\left(u_{h}-\tilde{u}, \varphi\right)=\mathscr{B}\left(u-\tilde{u}, \phi_{h}\right) .
$$

Let us define the interpolant by requiring

$$
\begin{gathered}
\int_{T}(u-\tilde{u}) v d x d t=0, \quad v \in P_{k-1}(T)(\text { if } k=1), \\
\int_{l}(u-\tilde{u}) v d x=0, \quad v \in P_{k}(l), T \in \mathscr{C}^{h},
\end{gathered}
$$

where $l$ is the side of $T$ which is parallel to the $x$-axis. It is easy to see that $\tilde{u}$ is uniquely defined so far as $u \in W^{1,1}$. Applying the Bramble-Hilbert lemma [2] we obtain by standard reasoning the interpolation error estimates

$$
\begin{aligned}
\|u-\tilde{u}\|_{p, T} & \leqslant C h^{k+1}|u|_{W^{k+1, p}(T)}, \\
\|u-\tilde{u}\|_{p, \partial T} & \leqslant C h^{k+1-1 / p}|u|_{W^{k+1, p}(T)}, \quad 1 \leqslant p \leqslant \infty,
\end{aligned}
$$

where $C$ depends only on $\kappa$ and $k$. 
Now by (2.6) and (4.11a),

$$
\begin{aligned}
\mathscr{B}\left(u-\tilde{u}, \phi_{h}\right)= & \int_{\left(\Gamma_{n} \cap \bar{\Omega}\right) \backslash \partial \Omega_{-}}(u-\tilde{u})^{-}\left(\phi_{h}^{-}-\phi_{h}^{+}\right) \nu \cdot \beta d s \\
& +\int_{\Omega} a(u-\tilde{u}) \phi_{h} d x d t,
\end{aligned}
$$

where $\Gamma_{n}$ is as in (4.2). Applying on the right side the Hölder inequality, (4.9) and (4.11), and recalling (4.10), we have

$$
\int_{\Omega}\left(u_{h}-\tilde{u}\right) \varphi d x d t \leqslant C M^{2}\left(h^{k+1 / 2}+\|a\|_{\infty, \Omega} h^{k+1}\right)|u|_{W^{2, p}(\Omega)}\|\varphi\|_{q, \Omega},
$$

where $1 / p+1 / q=1$. Since this is valid for any $\varphi$ such that $\varphi_{\mid \Omega} \in L_{q}(\Omega), 1 \leqslant q \leqslant$ $\infty$, and since $\|a\|_{\infty, \Omega} h^{1 / 2} \leqslant C$, by our assumptions, it follows that

$$
\left\|u_{h}-\tilde{u}\right\|_{p, \Omega} \leqslant C M^{2} h^{k+1 / 2}|u|_{W^{2 . p}(\Omega)}, \quad 1 \leqslant p \leqslant \infty .
$$

Recalling the estimate for $\|u-\tilde{u}\|_{p, \Omega}$ and using the triangle inequality one obtains the asserted estimate for $\left\|u-u_{h}\right\|_{p, \Omega}$.

The remaining estimates are proved in a similar manner by first introducing a function $\varphi \in L_{1}(\mathbf{R})$, replacing the right-hand side of (4.8) by

$$
\int_{\partial T \cap\{t=j h\}} \varphi v d x, \quad T \in \mathscr{M}^{h}, T \subset \Omega_{n},
$$

and then proceeding as above. We omit the details.

We consider finally a more practical situation where the triangulation $\mathscr{C}^{h}$ in (2.1) is only piecewise uniform.

THEOREM 4.3. Let $\mathscr{C}^{h}$ be a piecewise-uniform triangulation of $\Omega$ generated by $\mathscr{C}$ and let otherwise the assumptions of Theorem 4.2 hold. Then if $u \in W^{k+1, p}(\Omega)$, $1 \leqslant p \leqslant \infty$, we have the error estimate

$$
\left\|u-u_{h}\right\|_{p, \Omega} \leqslant \begin{cases}C h^{k+1-1 / p}|u|_{W^{k+1 . p}(\Omega)}, & \text { if } 1 \leqslant p \leqslant 2, \\ C h^{k+1 / 2}|u|_{W^{k+1 . p}(\Omega)}, & \text { if } 2 \leqslant p \leqslant \infty,\end{cases}
$$

where $C$ depends on $\mathscr{C}$ and on $\|a\|_{\infty, \Omega}$.

Proof. For $K \in \mathscr{C}$, let $u_{h}^{K}$ be the local discontinuous Galerkin approximation to $u$ on $K$ defined by (1.2) for $T \in \mathscr{C}^{h}, T \subset K$, with $u_{h}^{-}$replaced by $u$ on $\partial K_{-}$. Then $u_{\left.h\right|_{K}}=u_{h}^{K}+v_{h}^{K}$, where $v_{h}^{K}$ is the discontinuous Galerkin solution to the problem

$$
\begin{array}{ll}
\varphi_{\beta}+a \varphi=0 & \text { in } K, \\
\varphi=u_{h}^{-}-u & \text { on } \partial K_{-} .
\end{array}
$$

Applying Theorem 4.2, we have

$$
\begin{aligned}
\left\|u-u_{h}\right\|_{p, K} & \leqslant\left\|u-u_{h}^{K}\right\|_{p, K}+\left\|v_{h}^{K}\right\|_{p, K} \\
& \leqslant C h^{k+1 / 2}|u|_{W^{k+1, p}(\Omega)}+\left\|v_{h}^{K}\right\|_{p, K}, \quad 1 \leqslant p \leqslant \infty .
\end{aligned}
$$

Assume for a moment the further estimates

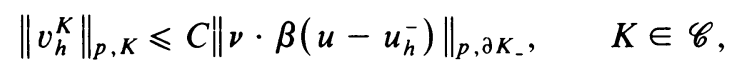


and

$$
\left\|\nu \cdot \beta\left(u-u_{h}^{-}\right)\right\|_{p, \partial K_{+}} \leqslant C h^{k+1 / 2}|u|_{W^{k+1, p}(K)}, \quad K \in \mathscr{C} .
$$

Then the repeated use of (4.13) through (4.15) gives

$$
\left\|u-u_{h}\right\|_{p, \Omega} \leqslant C h^{k+1 / 2}|u|_{W^{k+1, p}(\Omega)} .
$$

Let us now consider the validity of (4.14) and (4.15). Assume first that $\partial K_{-}$ contains two sides of $K$ and denote by $w_{h}^{K}$ the approximate solution of (4.12) in the case $a=0$. Then it follows from Theorem 3.2, via an affine transformation, that

$$
\left\|w_{h}^{K}\right\|_{p, K} \leqslant C\left\|\nu \cdot \beta\left(u-u_{h}^{-}\right)\right\|_{p, \partial K_{-},}, \quad 1 \leqslant p \leqslant \infty .
$$

Further, applying Theorem 4.1, we easily see that

$$
\left\|v_{h}^{K}-w_{h}^{K}\right\|_{p, K} \leqslant C\left\|a w_{h}^{K}\right\|_{p, K}, \quad 1 \leqslant p \leqslant \infty,
$$

so combining these estimates we conclude that (4.14) is valid for any $p$ if $\partial K_{-}$

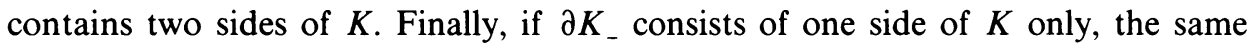
result can be read directly from Theorem 4.2 .

If $p=2$, the estimate (4.15) is valid by Theorem 2.1, and for $p=\infty$ (4.15) follows from Theorem 4.2. Thus, by interpolation [1], (4.15) and (4.16) are true in the range $2 \leqslant p \leqslant \infty$. In the range $1 \leqslant p<2$, however, (4.15) cannot hold, since it would in this case violate the approximation properties of piecewise-polynomial spaces. Thus, we need a different reasoning if $p<2$.

Consider the case $p=1$. Let $\varphi \in L_{\infty}(\Omega)$ and $\phi_{h} \in V_{h}$ be defined by (2.8). By Theorem 3.2 and by Theorem 4.1, we have

$$
\left\|\phi_{h}\right\|_{\infty, K} \leqslant C\left(\|\varphi\|_{\infty, K}+\left\|\phi_{h}^{+}\right\|_{\infty, \partial K_{+}}\right), \quad K \in \mathscr{C},
$$

and so, by iteration,

$$
\left\|\phi_{h}\right\|_{\infty, \Omega} \leqslant C\|\varphi\|_{\infty, \Omega} .
$$

Using now an argument from the proof of Theorem 4.2, with (4.9) replaced by the weaker estimate (4.17), it follows that

$$
\left\|u-u_{h}\right\|_{1, \Omega} \leqslant C h^{k}|u|_{W^{k+1.1}(\Omega)} .
$$

Upon interpolating between this estimate and the estimate (4.16) with $p=2$, and recalling that (4.16) holds for $p \geqslant 2$, the proof is finally complete.

Remark. In the proof of Theorems 4.2 and 4.3 we have used the increased stability of the discontinuous Galerkin method as established by the fundamental inequality (3.9). It is possible to state this stability inequality in a form analogous to that given in Theorem 2.1. For example, if $g=0$ in (1.1) and the triangulation $\mathscr{C}^{h}$ is uniform, we have (in the cases $k=0$ and $k=1$ ) that

$$
\left|u_{h}\right|_{h, \beta, p} \leqslant C h^{-1 / 2}\|f\|_{p, \Omega}, \quad 1 \leqslant p \leqslant \infty,
$$

where

$$
|v|_{h, \beta, p}=\left\{\sum_{T \in \mathscr{C}^{h}}\left\|v_{\beta}\right\|_{p, T}^{p}+h^{-p+1}\left\|\nu \cdot \beta\left(v^{+}-v^{-}\right)\right\|_{p, \Gamma_{h}}^{p}\right\}^{1 / p} .
$$


For a piecewise-uniform triangulation, the estimate remains valid in the range $1 \leqslant p \leqslant 2$, but ceases to be valid in the range $2<p \leqslant \infty$, as can be shown by a counterexample. In particular, if $p=\infty$ one can show that the estimate

$$
\left|u_{h}\right|_{h, \beta, \infty} \leqslant C h^{-1}\|f\|_{\infty, \Omega}
$$

is optimal. Since this follows already from the $L_{\infty}$-stability estimate using an inverse inequality, we see that in this case the smoothing property of the discontinuous Galerkin method is lost.

Department of Mathematics

Chalmers University of Technology

S-41296 Göteborg, Sweden

Institute of Mathematics

Helsinki University of Technology

SF-02150 Espoo 15, Finland

1. J. Bergh \& J. LOfSTROM, Interpolation Spaces, Springer-Verlag, Berlin and New York, 1976.

2. J. H. Bramble \& S. R. Hilbert, "Estimation of linear functionals on Sobolev spaces with application to Fourier transforms and spline interpolation," SIAM J. Numer. Anal., v. 7, 1970, pp. $112-124$.

3. Ph. Brenner \& V. Thomee, "Estimates near discontinuities for some difference schemes," Math. Scand., v. 28, 1971 , pp. 329-340.

4. Ph. Brenner, V. Thomé \& L. Wahlbin, Besov Spaces and Applications to Difference Methods for Initial Value Problems, Lecture Notes in Math., Vol. 434, Springer-Verlag, Berlin and New York, 1975.

5. G. Hedstrom, "The rate of convergence of some difference schemes," SIAM J. Numer. Anal., v. 5, 1968, pp. $363-406$.

6. C Johnson, U. Nävert \& J. Pitkäranta, "Finite element methods for linear hyperbolic problems," Comput. Methods Appl. Mech. Engrg., v. 45, 1984, pp. 285-312.

7. C. Johnson \& J. PitkÄranta, "Convergence of a fully discrete scheme for two-dimensional neutron transport," SIAM J. Numer. Anal., v. 20, 1983, pp. 951-966.

8. C. Johnson \& Mingyoung HuAng, "An analysis of the discontinuous Galerkin method for Friedrichs systems." (To appear.)

9. P. Lesaint \& P.-A. Raviart, "On a finite element method for solving the neutron transport equation," in Mathematical Aspects of Finite Elements in Partial Differential Equations (C. de Boor, ed.), Academic Press, New York, 1974.

10. U. NäVERT, A Finite Element Method for Convection-Diffusion Problems, Thesis, Department of Computer Science, Chalmers University of Technology, 1982.

11. W. H. Reed, T. R. Hill, F. W. Brinkley \& K. D. Lathrop, TRIDENT, a Two-Dimensional, Multigroup, Triangular Mesh, Explicit Neutron Transport Code, LA-6735-MS, Los Alamos Scientific Laboratory, 1977. 